\title{
On metal-deficient barium stars and their link with yellow symbiotic stars ${ }^{\star, \star \star}$
}

\author{
A. Jorissen ${ }^{1, \star \star \star}$, L. Začs ${ }^{2}$, S. Udry ${ }^{3}$, H. Lindgren ${ }^{4}$, and F. A. Musaev ${ }^{5,6,7}$ \\ 1 Institut d'Astronomie et d'Astrophysique, Université Libre de Bruxelles, CP 226, Boulevard du Triomphe, \\ 1050 Bruxelles, Belgium \\ e-mail: ajorisse@astro.ulb.ac.be \\ 2 Institute of Atomic Physics and Spectroscopy, University of Latvia, Raiņa bulvāris 19, Rīga, LV 1586 Latvia \\ 3 Observatoire de Genève, 1290 Sauverny, Suisse \\ 4 Lund Observatory, Box 43, 22100 Lund, Sweden \\ 5 Special Astrophysical Observatory and Isaac Newton Institute of Chile, SAO Branch, Nizhnij Arkhyz 369167, Russia \\ 6 ICAMER, National Academy of Sciences of Ukraine, 361605 Peak Terskol, Kabardino-Balkaria, Russia \\ 7 Shamakhy Astrophysical Observatory, National Academy of Sciences of Azerbaijan, Azerbaijan
}

Received 25 April 2005 / Accepted 20 May 2005

\section{ABSTRACT}

This paper addresses the question of why metal-deficient barium stars are not yellow symbiotic stars (YSyS). Samples of (suspected) metaldeficient barium (mdBa) stars and YSyS have been collected from the literature, and their properties reviewed. It appears in particular that the barium nature of the suspected $\mathrm{mdBa}$ stars needs to be ascertained by detailed abundance analyses. Abundances are therefore derived for two of them, HD 139409 and HD 148897, which reveal that HD 148897 should not be considered a barium star. HD 139409 is a mild barium star, with overabundances observed only for elements belonging to the first s-process peak ( $\mathrm{Y}$ and $\mathrm{Zr}$ ). It is only moderately metal-poor $([\mathrm{Fe} / \mathrm{H}]=-0.4)$. The evidence for binarity among mdBa stars is then reviewed, using three different methods: (i) radial-velocity variations (from CORAVEL observations), (ii) Hipparcos astrometric data, and (iii) a method based on the comparison between the Hipparcos and Tycho-2 proper motions. An orbit is obtained for HIP 55852, whereas evidence for the (so far unknown) binary nature of HIP 34795 , HIP 76605, HIP 97874 and HIP 107478 is presented. No conclusion regarding the binary nature of HIP 11595, HIP 25161 could be reached. Two stars with no evidence for binarity whatsoever (HIP 58596 and BD $+3^{\circ} 2688$ ) are candidates low-metallicity thermally-pulsing asymptotic giant branch stars, as inferred from their large luminosities. The reason why mdBa stars are not YSyS is suggested to lie in their different orbital period distributions: mdBa stars have on average longer orbital periods than YSyS, and hence their companion accretes matter at a lower rate, for a given mass loss rate of the giant star. The definite validation of this explanation should nevertheless await the determination of the orbital periods for the many mdBa stars still lacking periods, in order to make the comparison more significant.

Key words. binaries: symbiotic - stars: abundances - stars: AGB and post-AGB - binaries: spectroscopic

\section{The problem}

Our understanding of the link between chemically-peculiar red giants like barium stars or $\mathrm{CH}$ stars, and yellow symbiotic stars (YSyS) has made substantial progress in the last decade (see the reviews by Jorissen 2003a,b), mainly with the realisation that likely all yellow symbiotics (i.e., involving a giant of spectral type $\mathrm{G}$ or $\mathrm{K}$ as primary component) involve barium stars (Smith et al. 1996, 1997;

* Based on observations carried out at the European Southern Observatory (ESO, La Silla, Chile) and with the 1-m Swiss telescope at the Haute-Provence Observatory.

$\star \star$ Table 7 is only available in electronic form at http://www. edpsciences.org

$\star \star \star$ Senior Research Associate, FNRS, Belgium.
Pereira \& Porto de Mello 1997; Pereira et al. 1998). The metaldeficient nature of the giant is a key factor, because it implies a rather large luminosity for the giant. Since evolutionary tracks of metal-deficient stars are shifted towards the blue, metaldeficient giants of spectral type K must lie on the upper part of the (asymptotic) giant branch (see Fig. 11 of Smith et al. 1996), where they suffer strong mass loss. If such metal-deficient K giants are in binary systems, their strong wind will interact with the companion and trigger symbiotic activity.

The other facet of this problem, namely whether all metaldeficient barium stars are symbiotic stars, is not yet fully answered. The present paper offers a first step in that direction.

We have collected a list of candidate metal-deficient barium stars and have assembled new observations to check (i) whether 
Table 1. The barium syndrome among YSyS. The spectral type of the cool component is taken from Mürset \& Schmid (1999), or references therein. In column labelled "nebula", "y" means that an optical nebula has been detected, and "PN" that, based on its emission line spectrum, the star has traditionally been included in planetary nebulae catalogues, even though no optical nebula may be visible. The column labelled " $P$ " lists the orbital period, from Mürset \& Schmid (1999).

\begin{tabular}{|c|c|c|c|c|c|c|c|c|c|}
\hline Name & Sp. typ. & {$[\mathrm{Fe} / \mathrm{H}]$} & $\begin{array}{c}V_{r} \\
\left(\mathrm{~km} \mathrm{~s}^{-1}\right) \\
\end{array}$ & $\begin{array}{c}b \\
\left({ }^{\circ}\right)\end{array}$ & {$[\mathrm{Ba} / \mathrm{Fe}]$} & $\begin{array}{l}V \sin i \\
\left(\mathrm{~km} \mathrm{~s}^{-1}\right)\end{array}$ & Nebula & $\begin{array}{l}P \\
\text { (d) }\end{array}$ & Ref. \\
\hline \multicolumn{10}{|c|}{ d'-type } \\
\hline V417 Cen & G8-K2 & $\sim 0.0$ & & -1 & 0.5 & 70 & $\mathrm{y}$ & 247 & $(5,11)$ \\
\hline \multicolumn{10}{|l|}{$=$ Cn $1-1$} \\
\hline AS 201 & G5 & 0.07 & & +7 & 0.63 & 25 & $\mathrm{y}$ & - & $(12,17)$ \\
\hline \multicolumn{10}{|l|}{$=\mathrm{M} 1-2$} \\
\hline St $\mathrm{H}_{\alpha} 190$ & G5 & 0.0 & $\sim 10$ & -35 & $\sim 0.5$ & 100 & bip. outf. & - & $(10,13)$ \\
\hline Wray 157 & G5 & $?$ & & & & & & & \\
\hline Hen 1591 & $<\mathrm{K} 4$ & $?$ & & & & & & & \\
\hline \multicolumn{10}{|c|}{ s-type } \\
\hline UKS Ce-1 & $\mathrm{C} 4,5 \mathrm{Jch}$ & $?$ & +20 & +20 & $>0$ & & & - & (6) \\
\hline S 32 & $\mathrm{C} 1,1 \mathrm{CH}$ & $?$ & +325 & -30 & $>0$ & & & 612 & $(6,14)$ \\
\hline Hen 2-467 & K0 & -1.1 & -109 & -12 & +0.8 & & $\mathrm{n}$ & 478 & $(4,16)$ \\
\hline \multirow[t]{2}{*}{$\mathrm{BD}-21^{\circ} 3873$} & $\mathrm{~K} 2$ & -1.1 & +204 & +37 & +0.5 & & $\mathrm{n}$ & 282 & $(3,15,16)$ \\
\hline & & -1.3 & & & +0.3 & & & & (9) \\
\hline AG Dra & $\mathrm{K} 2$ & -1.3 & -148 & +41 & +0.5 & & $\mathrm{n}$ & 554 & $(8,16)$ \\
\hline $\mathrm{CD}-43^{\circ} 14304$ & K7 & -1.4 & +27 & -41 & $?$ & & & 1448 & $(7,18)$ \\
\hline \multicolumn{10}{|c|}{ Chemical evolution of the Galaxy } \\
\hline & & -1.0 & & & $<0.2$ & & & & (1) \\
\hline
\end{tabular}

References: (1) Edvardsson et al. 1993, A\&A, 275, 101; (2) Grauer \& Bond 1981, PASP, 93, 630; (3) Pereira et al. 1997, AJ, 114, 2128; (4) Pereira et al. 1998, AJ, 116, 1977; (5) Pereira et al. 2003, in Symbiotic stars probing stellar evolution, ed. R. L. M. Corradi, J. Mikołajewska, \& T. J. Mahoney, Astron. Soc. Pacific Conf. Ser. (San Francisco), 85; (6) Schmid 1994, A\&A, 284, 156; (7) Schmid et al. 1998, A\&A, 329, 986; (8) Smith et al. 1996, A\&A, 315, 179; (9) Smith et al. 1997, A\&A, 324, 97; (10) Smith et al. 2001, ApJ, 556, L55; (11) Van Winckel et al. 1994, A\&A, 285, 241; (12) Schwarz 1991, A\&A, 243, 469; (13) Munari et al. 2001, A\&A, 369, L1; (14) Schmid \& Nussbaumer 1993, A\&A, 268, 159; (15) Munari \& Patat 1993, A\&A, 277, 195; (16) Corradi et al. 1999, A\&A, 343, 841; (17) Pereira et al. 2005, A\&A, 429, 993; (18) The metallicity is from Pereira, priv. comm.

these stars are binaries, (ii) whether they are barium stars and (iii) whether they exhibit symbiotic activity.

\section{The samples}

Before discussing metal-deficient barium stars, it is useful to first summarize the properties of YSyS, to which metaldeficient barium stars may be compared.

\subsection{Yellow symbiotic stars}

All known YSyS are listed in Table 1, which shows that all the stars studied so far exhibit the barium syndrome. YSyS with a stellar infrared continuum (s-type, as opposed to the dusty d'-type; see below) are clearly metal-deficient objects, as revealed by their low metallicities and high space velocities (CD $-43^{\circ} 14304$ may be an exception; however, it is of spectral type K7, and should perhaps not be included in the family of YSyS). The presence of the barium syndrome among a family of binary metal-deficient stars fully supports the commonly accepted hypothesis that the s-process is more efficient at low metallicities (Clayton 1988; Jorissen 2003a). s-Type YSyS, with their metallicities lower than classical barium stars, may be expected to be, on average, more luminous than the latter (see Fig. 11 of Smith et al. 1996, comparing the luminosity function of Pop.I and Pop.II K giants). This is a direct consequence of the fact that evolutionary tracks shift towards the blue in the Hertzsprung-Russell (HR) diagram as metallicity decreases, as shown in Fig. 1b. Figure 1a confirms that the YSyS AG Dra and BD $-21^{\circ} 3873$ are indeed more luminous than classical barium stars. This difference in the average luminosity - and hence mass-loss rate - of the two populations thus explains why YSyS, despite hosting a K giant, exhibit symbiotic activity whereas barium stars do not. The larger mass-loss rates for the cool components of s-type YSyS - as compared to Ba stars - may be inferred from the comparison of their IRAS [12]-[25] color indices, which reflect the amount of dust present in the system: $([12]-[25])_{\mathrm{Ba}}<0.1$, as compared to 0.45 

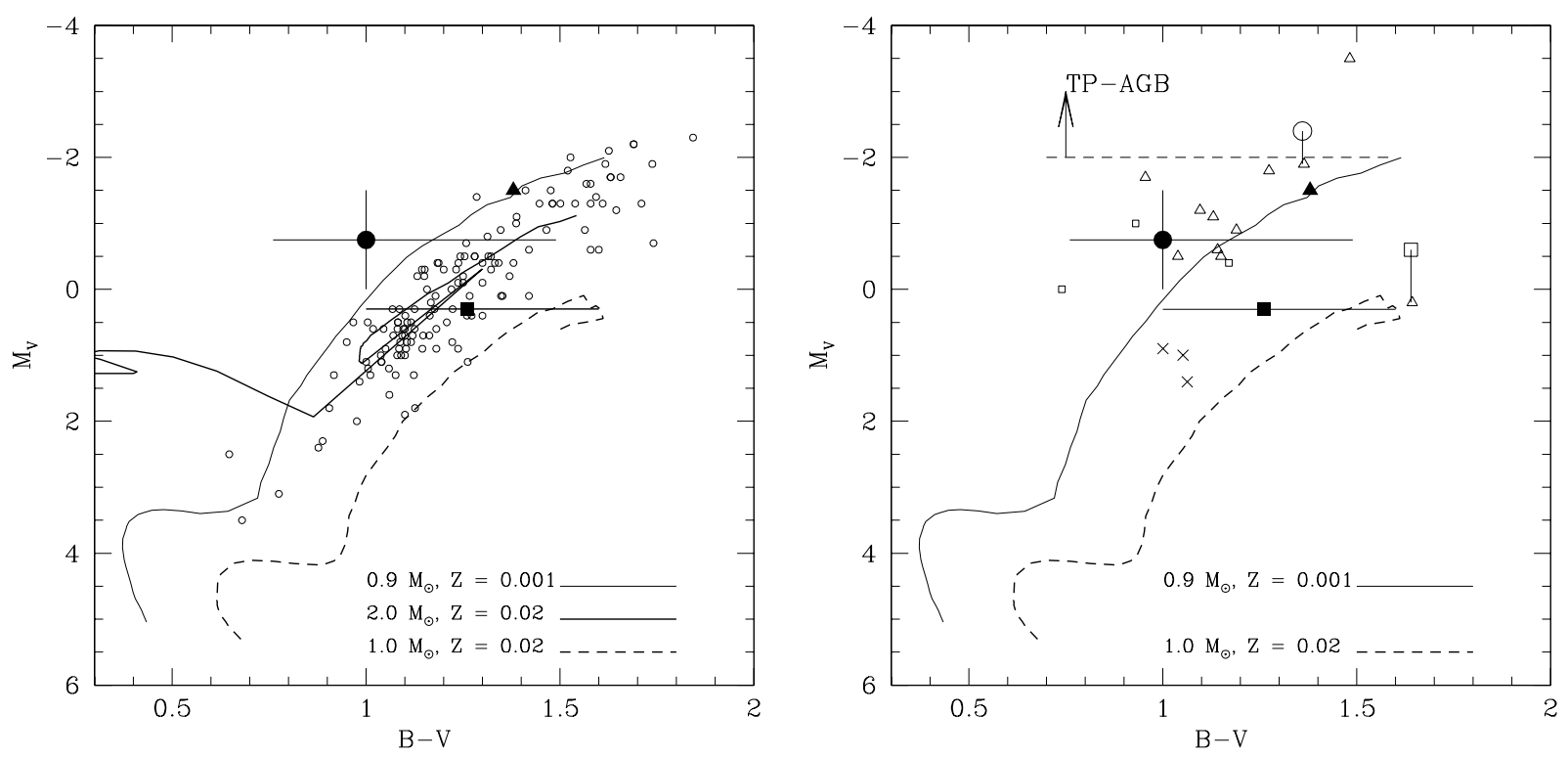

Fig. 1. Left panel a): evolutionary tracks of Schaller et al. (1992) compared with the locations of classical barium stars (stars labelled "G" in Mennessier et al. (1997); open dots) and the yellow SyS AG Dra (filled circle), BD -21 3873 (filled triangle) and Hen 2-467 (filled square). The bolometric magnitudes were taken from the references listed in Table 1. These bolometric magnitudes were combined with bolometric corrections from Bessell et al. (1998) and $B-V$ indices from Munari et al. (1992) and Munari \& Buson (1992) to yield the absolute visual magnitudes. Right panel b): same as a) but for YSyS (filled symbols as in the left panel) and metal-deficient barium stars [open triangles: stars flagged as "H" by Mennessier et al. (1997); crosses: CH stars also flagged as "H" by Mennessier et al. (1997); small open squares: additional metal-deficient, s-process-rich stars from Table 2; open circle: HD 104340, large open square: HD 206983 from Junqueira \& Pereira (2001)]. The dashed horizontal line represents the luminosity $\left(M_{\mathrm{bol}}=-3\right.$, corresponding to $\left.M_{\mathrm{V}} \sim-2\right)$ at the first thermal pulse in a $1 M_{\odot}$ AGB star of metallicity $[\mathrm{Fe} / \mathrm{H}]=-1.8$ according to Lattanzio (1991).

for AG Dra (Smith et al. 1996). Mürset et al. (1991) and Drake et al. (1987) provide direct measurements (or upper limits) for the mass loss rates of AG Dra and of Ba stars, respectively, which confirm the above conclusion.

YSyS with a dusty infrared continuum (d'-type; Allen 1982; Schmid \& Nussbaumer 1993) differ from their s-type counterparts in several respects (Table 1): they host a complex circumstellar environment (including cool dust, bipolar outflows, extended optical nebulae or emission-line spectra closely resembling those of planetary nebulae), the cool components have early spectral types ( $\mathrm{F}$ to early $\mathrm{K}$ ), they are often fast rotators (with the possible exception of M 1-2 = V471 Per; Grauer \& Bond 1981) and, finally, they belong to the galactic disk unlike s-type YSyS which belong to the halo.

All these arguments suggest that the hot component in d'-type SyS has just evolved from the AGB to the WD stage. The rather cool dust (Schmid \& Nussbaumer 1993) is a relic from the mass lost by the AGB star. The optical nebulae observed in d'-type SyS are most likely genuine planetary nebulae rather than the nebulae associated with the ionized wind of the cool component (Corradi et al. 1999). This is especially clear for AS 201 which actually hosts two nebulae (Schwarz 1991): a large fossil planetary nebula detected by direct imaging, and a small nebula formed in the wind of the current cool component. Finally, the rapid rotation of the cool component has likely been caused by spin accretion from the former AGB wind like in WIRRING systems (Jeffries \& Stevens 1996; Jorissen 2003b). The fact that the cool star has not yet been slowed down by magnetic braking is another indication that the mass transfer occurred fairly recently (Theuns et al. 1996). Corradi \& Schwarz (1997) obtained 4000 y for the age of the nebula around AS 201, and 40000 y for V417 Cen.

\subsection{Metal-deficient barium stars}

Metal-deficient barium stars (with metallicities in the range -1.1 to -1.8 , comparable to that of YSyS) were identified by Luck \& Bond (1991), Mennessier et al. (1997) and Začs et al. (2000), and occupy the same region of the HR diagram as YSyS (Fig. 1b). The question thus arises why metal-deficient barium stars are not YSyS. Different answers must be sought, depending upon their absolute visual magnitudes $M_{\mathrm{V}}$. The most luminous systems, with $M_{\mathrm{V}}<-2$, are likely located on the thermally-pulsing AGB, so that their Ba syndrome may be explained by internal nucleosynthesis. They thus should not be binaries, and therefore cannot be YSyS! HD 104340 (open circle in Fig. 1b), a metal-deficient Ba star studied by Junqueira \& Pereira (2001), and BD $+03^{\circ} 2688$ (Table 2) provide good illustrations of this situation, since they both lie above the TP-AGB threshold and CORAVEL radial-velocity measurements spanning several years do not reveal any clear orbital motion (Figs. 9 and 10, as well as Sect. 4).

The less luminous and warmest among metal-deficient Ba stars, clumping around $M_{\mathrm{V}} \sim+1$ in the HR diagram, are also sometimes classified as $\mathrm{CH}$ stars (crosses in Fig. 1b). They are not losing mass at a large enough rate to trigger any symbiotic activity, as revealed by their small [12]-[25] color indices $(<0.3$; Smith et al. 1996). 
Table 2. Stars classified as metal-deficient barium stars by Mennessier et al. (1997). The absolute magnitude $M_{V}$ is a maximum likelihood estimate obtained by Mennessier et al. (1997), except for the additional stars where it is derived from a straight inversion of the Hipparcos parallax ( $\left.\mathrm{BD}+75^{\circ} 348\right)$, from a spectroscopic estimate of gravity and an educated guess for the mass (BD $\left.+3^{\circ} 2688\right)$ or from a fit to the M 92 isochrone (CS stars). The column labelled "Ba" indicates whether detailed chemical analyses have confirmed the Ba nature of the star.

\begin{tabular}{|c|c|c|c|c|c|c|c|c|}
\hline HIP & $\mathrm{HD} / \mathrm{DM}$ & $M_{V}$ & $B-V$ & {$[\mathrm{Fe} / \mathrm{H}]$} & Ref. & $\mathrm{Ba}$ & Ref. & Rem. \\
\hline 4347 & 5424 & -0.6 & 1.14 & & & & & \\
\hline 11595 & 15589 & -0.5 & 1.15 & -0.7 & 9 & & & \\
\hline 25161 & $-27^{\circ} 2233$ & -1.1 & 1.13 & & & & & \\
\hline 29740 & 43389 & -3.5 & 1.48 & & & & & \\
\hline 34795 & 55496 & -1.7 & 0.96 & -1.55 & 5 & $\mathrm{y}$ & 5 & \\
\hline 58596 & 104340 & -1.9 & 1.36 & -1.72 & 3 & $\mathrm{y}$ & 3 & \\
\hline 69834 & 123396 & -0.9 & 1.19 & & & & & \\
\hline 76605 & 139409 & -0.5 & 1.04 & -0.42 & 2 & mild & 2 & $M_{V}=1.5$ is derived from spectroscopic $\log g$ \\
\hline \multirow[t]{3}{*}{80843} & 148897 & -1.8 & 1.27 & -1.0 & 2 & $\mathbf{n}$ & 2 & \\
\hline & & & & -0.62 & 7 & & & \\
\hline & & & & -1.16 & 8 & & & \\
\hline 97874 & 187762 & -1.2 & 1.10 & & & & & \\
\hline 107478 & 206983 & 0.2 & 1.64 & -1.43 & 3 & $\mathrm{y}$ & 3 & \\
\hline \multicolumn{9}{|c|}{ Additional stars (from refs. (1), (5) and (10)) } \\
\hline 43042 & $+75^{\circ} 348$ & $-0.4:$ & 1.17 & -0.8 & 1 & $\mathrm{y}$ & 1 & \\
\hline \multirow[t]{4}{*}{55852} & $+4^{\circ} 2466$ & $?$ & 0.60 & -1.85 & 5,11 & $\mathrm{y}$ & 4,5 & \\
\hline & $+3^{\circ} 2688$ & -5.0 & 1.22 & -1.42 & 5,11 & $\mathrm{y}$ & 5 & \\
\hline & CS 22942-019 & 0 & 0.74 & -2.67 & 10 & $\mathrm{y}$ & 10 & \\
\hline & CS 22948-027 & -1 & 0.93 & -2.60 & 10 & $\mathrm{y}$ & 10 & \\
\hline
\end{tabular}

References to the table: (1) Začs et al. (2000); (2) this work; (3) Junqueira \& Pereira (2001); (4) Burris et al. (2000); (5) Luck \& Bond (1991); (6) Jorissen et al. (1998); (7) Kyröläinen et al. (1986); (8) Luck (1991); (9) Barbuy et al. (1992); (10) Preston \& Sneden (2001); (11) B - V from the Tycho-2 catalog (Høg et al. 2000b).

Finally, at intermediate luminosities $\left(-2 \leq M_{\mathrm{V}} \leq+1\right)$ where YSyS are located, metal-deficient Ba stars are not luminous enough to be TP-AGB (hence they should be binaries), but yet their mass loss rates must be large enough to trigger symbiotic activity (provided that the orbital separation is not too large, since it is the mass accretion rate by the compact companion which is in fact the key parameter; see Sect. 5 and Jorissen 2003a). It is thus of great interest to check (i) the Ba nature of those metal-deficient stars with intermediate luminosities, (ii) their binary nature, and (iii) their suspected symbiotic activity. The first two issues are addressed in Sects. 3 and 4, respectively.

As far as a possible symbiotic activity is concerned, there is no indication from their photometric $U-B$ and $B-V$ indices that the metal-deficient stars in Table 2 have a strong blue continuum which could betray their symbiotic nature. It is thus very likely that none among these stars is a full-fledged symbiotic star. No signature of weak symbiotic activity (of the kind exhibited by some binary S stars; see Fig. 14 and Van Eck \& Jorissen 2002) is observed in the $\mathrm{H}_{\alpha}$ line profile either (Fig. 2).

\section{Abundances}

The classification of the stars in Table 2 as metal-deficient Ba stars is subject to caution, as it does not rely on spectroscopic abundance analyses, but rather on a maximumlikelihood assignment based on kinematic, spatial and luminosity properties (Mennessier et al. 1997) for barium stars from the list of Lü (1991). Nevertheless, when a metallicity determination is available, it confirms the metal-deficient nature of the object (see Table 2). HD 139409 is an exception, though, since detailed spectral analyses reveal that it is neither metalpoor nor strongly enriched in s-process elements (see below). It may nevertheless be hoped that the metal-deficient assignment made by Mennessier et al. (1997) is valid in all the other cases. Regarding the Ba nature of these stars, it is known that the Lü (1991) catalogue of barium stars, from which the sample of barium stars used by Mennessier et al. (1997) was drawn, is contaminated by many non-barium stars (Griffin \& Keenan 1992; Jorissen et al. 1996), especially among those stars having a Ba index smaller than 1 .

It would therefore in principle be necessary to re-evaluate the $\mathrm{Ba}$ nature of all the stars listed in Table 2. So far, spectra could be obtained for two of them, HD 139409 and HD 148897, which are discussed in detail in the present section. Of these, only HD 139409 appears to be a mild barium star, thus confirming the suspicion about the Lü (1991) catalogue expressed above.

\subsection{The case of HD 148897 and HD 139409}

HD 148897 (=HR 6152) has been tagged as a "likely marginal barium star" by Boyle \& McClure (1975) and as a marginal CH star by Vilhu et al. (1977). It therefore found its way into 


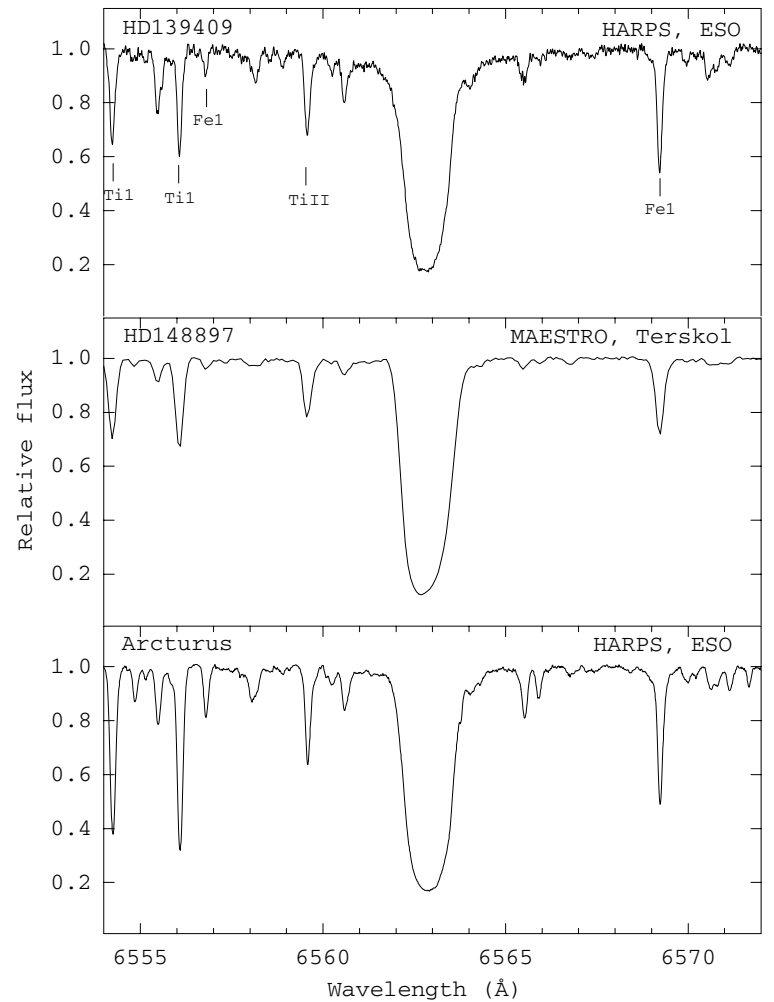

Fig. 2. $H_{\alpha}$ profiles for two candidate metal-deficient Ba stars (HD 139409 and HD 148897; see however the discussion in Sect. 3), compared to that of the $\mathrm{K}$ giant Arcturus. The wavelength scale has been corrected for the stellar radial velocity. The profiles show no indication of symbiotic activity.

the barium-star catalogs of Lü et al. (1983) and Lü (1991), as well as the catalog of $\mathrm{CH}$ and metal-deficient Ba stars of Šleivyte \& Bartkevičius (1990). The star was classified as G8.5III CN-2 Fe-1 CH-1 by Keenan \& McNeil (1989), and this classification as $\mathrm{CN}$ - and $\mathrm{CH}$-weak contradicts the earlier assignments.

A detailed abundance analysis has thus been performed to clarify the situation, and its results are compared with previous analyses by Kyröläinen et al. (1986) and Luck (1991) in Sect. 3.1.2.

HD 139409 (=HIP 76605) has been classified as a marginal barium star by MacConnell et al. (1972), as G5 III Ba1 by Yamashita \& Norimoto (1981) and as K0 III/II Ba 0.5 by Lü (1991).

\subsubsection{Observations}

A high-resolution spectrum of HD 148897 was obtained using the Coudé Matrix Echelle Spectrometer (MAESTRO; Musaev et al. 1999) delivering a resolving power of 45000 and installed on the 2-m Zeiss telescope of the Terskol Observatory (located in Northern Caucasus at an altitude of $3100 \mathrm{~m}$ ). The spectrometer is equipped with a Wright Instruments CCD detector with $1242 \times 1152$ pixels $(22.5 \times 22.5 \mu \mathrm{m})$. A total exposure of $1800 \mathrm{~s}$ was taken on February 18, 2003. The spectrum covers the range 365 to $1020 \mathrm{~nm}$ spread over 85 spectral orders.

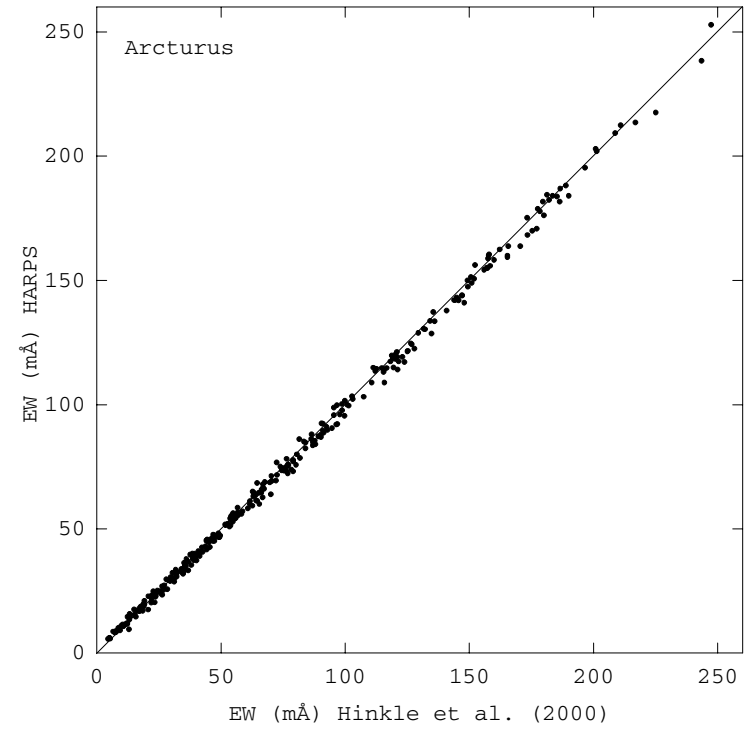

Fig. 3. Comparison of equivalent widths measured on the HARPS spectrum of Arcturus with those of the Arcturus spectral atlas (Hinkle et al. 2000), for the same set of lines as that measured in HD 139409.

A high-resolution spectrum of HD 139409 was obtained on the HARPS spectrograph (Mayor et al. 2003), delivering a resolution of 115000 and installed on the ESO La Silla $3.6 \mathrm{~m}$ telescope. A total exposure of $200 \mathrm{~s}$ was obtained by Xavier Bonfils on May 31, 2004. In order to check equivalent widths delivered by HARPS, a spectrum of the standard star Arcturus was obtained as well by Fabien Carrier. The HARPS spectra were reduced by the observers using standard pipeline processing. Equivalent widths for the same set of lines as those studied in HD 139409 have been measured by one of us (L.Z.) in the HARPS spectrum of Arcturus and compared to those from the Arcturus spectral atlas (Hinkle et al. 2000). The agreement between the two sets of equivalent widths is excellent (Fig. 3), thus qualifying HARPS for abundance analyses. Spectra around the $\lambda 614.172 \mathrm{~nm}$ Ba II line are shown in Fig. 4 for the two analyzed stars and for Arcturus (Hinkle et al. 2000).

\subsubsection{Reduction and analysis}

HD 148897

The reduction of the CCD frames (subtraction of bias, dark and scattered light, flat fielding, extraction of echelle orders and wavelength calibration) was performed with the DECH20T software (Galazutdinov 1992). More than 500 weak to medium-strong atomic lines, free of blends, were identified and their equivalent widths were measured with the DECH routines. The radial velocity was measured using a large number of symmetric absorption lines. The observed velocity has been brought to the heliocentric system by adding $+22.7 \mathrm{~km} \mathrm{~s}^{-1}$. The mean heliocentric radial velocity for HD 144897 was found to be $+16.7 \mathrm{~km} \mathrm{~s}^{-1}$. The atmospheric parameters for that star cannot be derived from photometry, since the standard temperature calibrations refer to stars of normal chemical composition. To obtain a colour-independent estimate of the temperature, a spectroscopic temperature has been derived from the 


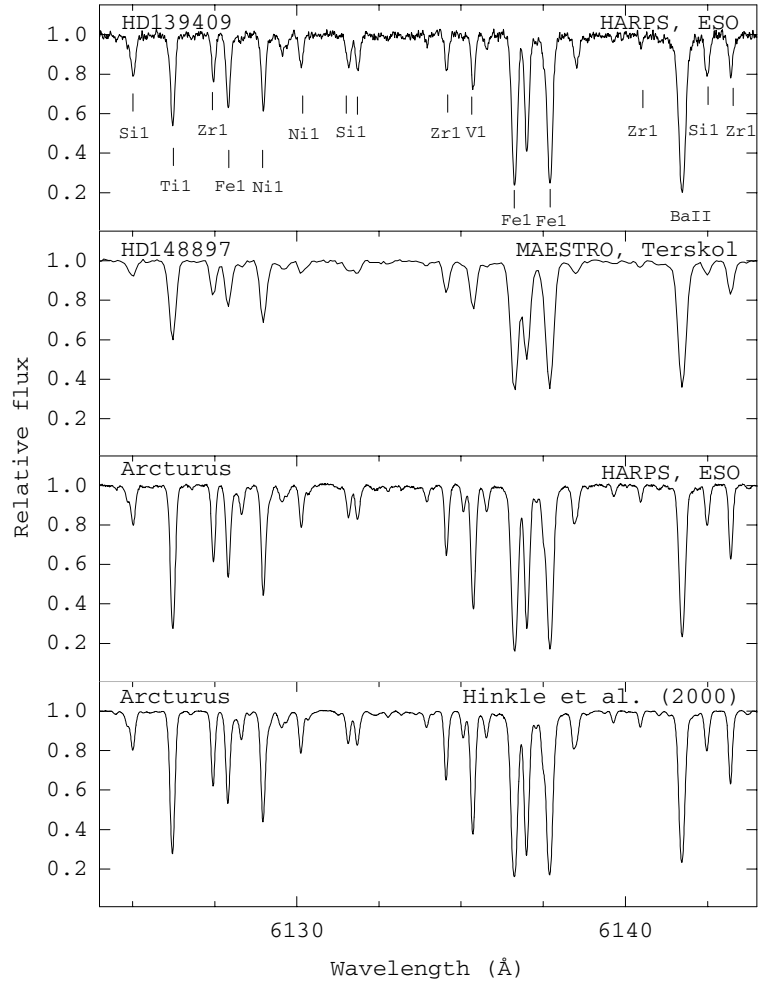

Fig. 4. The spectra of the target stars in the region of the Ba II line at $614.172 \mathrm{~nm}$. Measured lines are marked. Also shown is the spectrum of the standard star Arcturus (Hinkle et al. 2000).

excitation equilibrium of Fe I lines. The surface gravity $\log g$ was determined using the FeI/FeII ionization balance, whereas the microturbulent velocity $v_{\mathrm{t}}$ was derived by forcing the abundances of individual Fe I lines to be independent of the reduced equivalent width. The resulting atmospheric parameters for HD 148897 are as follows: $T_{\text {eff }}=4350 \mathrm{~K}, \log g=1.0(\mathrm{cgs})$, and $v_{\mathrm{t}}=2.0 \mathrm{~km} \mathrm{~s}^{-1}$. An independent determination of the surface gravity, using the above $T_{\text {eff }}$ value, Mennessier et al. (1997) absolute visual magnitude $\left(M_{V}=-1.8\right)$, a bolometric correction of -0.5 and a mass of $1 M_{\odot}$, yields $\log g=1.1$, in agreement with the adopted value (see Luck 1991, for a discussion of the discrepancy usually observed between the photometric and spectroscopic gravities). Comparison with atmospheric parameters from the literature is presented in Table 3.

The abundance analysis has been performed with the standard LTE line analysis program WIDTH9 developed by Kurucz. The model atmospheres were taken from Gustafsson et al. (1975). The synthetic spectra were generated using the spectral synthesis code STARSP (Tsymbal 1996). Oscillator strengths have been taken from the VALD database (Piskunov et al. 1995). The resulting abundances (normalized by the solarsystem abundances of Grevesse \& Sauval 1998) are listed in Table 3, from which it may be concluded that HD 148897 appears to be a rather typical metal-deficient star (McWilliam 1997), and should certainly not be considered as a (metaldeficient) barium star.

Interestingly enough, there are no indications whatsoever from the Hipparcos and Tycho data (applying the methods

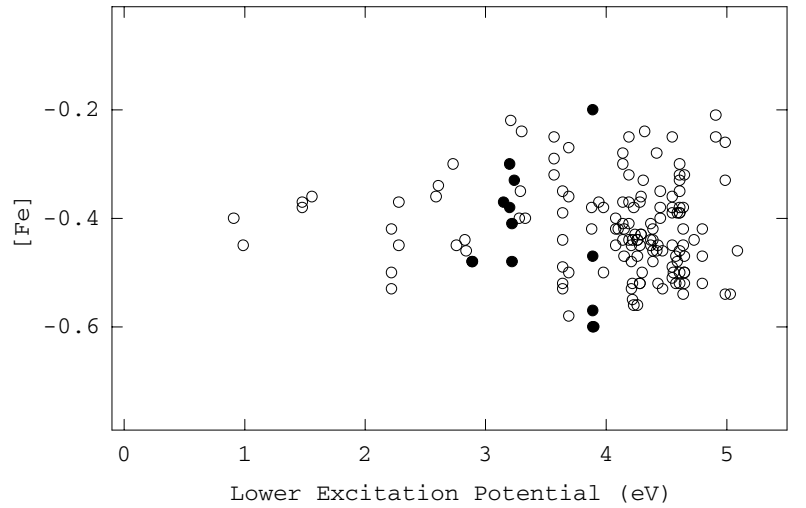

Fig. 5. The iron abundances derived from Fe I (open circles) and Fe II (filled circles) lines are displayed as a function of the excitation potential for the lower energy level of the line. The absence of a trend in these data is used to derive the spectroscopic temperature of HD 139409.

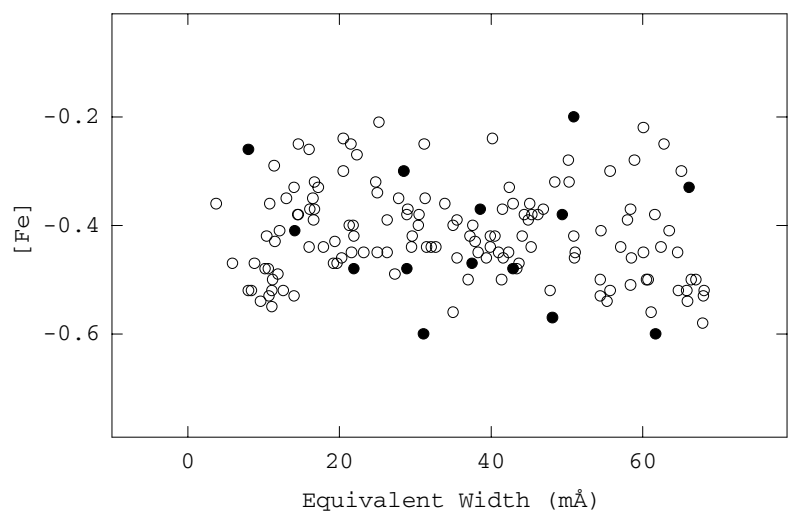

Fig. 6. The abundances derived from the Fe I (open circles) and Fe II (filled circles) lines are displayed as a function of the line equivalent widths. The absence of a trend in these data is used to derive the microturbulent velocity of HD 139409.

described in Sect. 4) that this star is binary, in agreement with the fact that it is not a barium star.

\section{HD 139409}

The effective temperature of HD 139409 has been derived from the excitation equilibrium of Fe I, Ti I and Cr I lines (see Fig. 5, for $\mathrm{Fe}$ ). The surface gravity $\log g$ was determined from the $\mathrm{Fe} \mathrm{I} / \mathrm{Fe}$ II ionization balance, and the microturbulent velocity by forcing the abundances of individual Fe I, Ti I and CrI lines to be independent of equivalent width (see Fig. 6, for Fe). Although spectroscopic gravity and temperature determinations in late-type, metal-deficient stars are probably affected by non-LTE effects, these effects remain small when $[\mathrm{Fe} / \mathrm{H}] \geq$ -1.0 (see, for example, Allende Prieto et al. 1999). The stellar parameters of Arcturus $([\mathrm{Fe} / \mathrm{H}]=-0.6, \log g=1.3)$ derived by the spectroscopic method (applied on the HARPS spectrum) are in good agreement indeed with those derived by other (nonspectroscopic) methods.

The resulting atmospheric parameters for HD 139409 are as follows: $T_{\text {eff }}=5000 \mathrm{~K}, \log g=2.8(\mathrm{cgs})$, and $v_{\mathrm{t}}=$ $2.0 \mathrm{~km} \mathrm{~s}^{-1}$. The spectroscopic gravity, combined with a mass of $1 M_{\odot}$, leads to $M_{V} \simeq+1.5$. Thus our calculations indicate 
Table 3. Averaged absolute abundances (in the scale where $\log \epsilon(\mathrm{H})=12$ ) and normalized abundances $[X / \mathrm{Fe}]$ for HD 148897 (relative to the Sun; Grevesse \& Sauval 1998). The standard deviations $\sigma$ and the number of lines used in the analysis $(n)$ are also given. Two sets of abundances from Luck (1991) are given: one is based on a "photometric $\log g$ " (derived from the absolute magnitude, the effective temperature and an estimate for the stellar mass) and the other on the "spectroscopic $\log g$ " ( $\mathrm{Fe} \mathrm{I/Fe} \mathrm{II} \mathrm{ionization} \mathrm{balance).}$

\begin{tabular}{|c|c|c|c|c|c|c|c|c|c|c|c|}
\hline \multirow[b]{2}{*}{$X$} & \multicolumn{5}{|c|}{$\begin{array}{c}\text { This work } \\
T_{\text {eff }}=4350 \mathrm{~K} \\
\log g=1.0 \\
v_{\mathrm{t}}=2.0 \mathrm{~km} \mathrm{~s}^{-1}\end{array}$} & \multicolumn{2}{|c|}{$\begin{array}{c}\text { Kiröläinen et al. (1986) } \\
T_{\text {eff }}=4360 \mathrm{~K} \\
\log g=1.5 \\
v_{\mathrm{t}}=2.6 \mathrm{~km} \mathrm{~s}^{-1} \\
\end{array}$} & \multicolumn{2}{|c|}{$\begin{array}{c}\text { Luck (1991) } \\
T_{\text {eff }}=4100 \mathrm{~K} \\
\text { phot. } \log g=1.6 \\
v_{\mathrm{t}}=1.7 \mathrm{~km} \mathrm{~s}^{-1} \\
\end{array}$} & \multicolumn{2}{|c|}{$\begin{array}{c}\text { Luck }(1991) \\
T_{\text {eff }}=4100 \mathrm{~K} \\
\text { spec. } \log g=0.1 \\
v_{\mathrm{t}}=1.7 \mathrm{~km} \mathrm{~s}^{-1}\end{array}$} \\
\hline & $\log \epsilon(X)$ & $\sigma$ & $n$ & {$[X / H]_{\odot}$} & {$\left[\frac{X_{\mathrm{I}, \mathrm{II}}}{\mathrm{Fe}_{\mathrm{I}, \mathrm{II}}}\right]_{\odot}$} & {$[X / H]_{\odot}$} & {$[X / \mathrm{Fe}]_{\odot}$} & {$[X / \mathrm{H}]_{\odot}$} & {$\left[\frac{X_{\mathrm{I}, \mathrm{II}}}{\mathrm{Fe}_{\mathrm{I}, \mathrm{II}}}\right]_{\odot}$} & {$[X / H]_{\odot}$} & {$\left[\frac{X_{\mathrm{I}, \mathrm{II}}}{\mathrm{Fe}_{\mathrm{I}, \mathrm{II}}}\right]_{\odot}$} \\
\hline O I & 8.22 & 0.20 & 4 & -0.61 & +0.41 & -0.58 & +0.04 & -0.50 & +0.50 & -1.10 & +0.06 \\
\hline $\mathrm{Na} \mathrm{I}$ & 5.10 & 0.08 & 6 & -1.23 & -0.21 & -1.11 & -0.49 & -1.36 & -0.36 & -1.26 & -0.10 \\
\hline $\mathrm{Mg} \mathrm{I}$ & 6.92 & 0.09 & 6 & -0.66 & +0.36 & -0.59 & +0.03 & -0.54 & +0.46 & -0.62 & +0.54 \\
\hline $\mathrm{Al} \mathrm{I}$ & 5.61 & 0.23 & 4 & -0.86 & +0.16 & -0.70 & -0.08 & -1.28 & -0.28 & -1.20 & -0.04 \\
\hline $\mathrm{Si} \mathrm{I}$ & 6.91 & 0.11 & 23 & -0.77 & +0.25 & -0.56 & +0.06 & -0.24 & +0.76 & -0.59 & +0.57 \\
\hline K I & 4.17 & & 1 & -0.95 & +0.07 & - & - & - & - & - & - \\
\hline $\mathrm{Ca} \mathrm{I}$ & 5.65 & 0.11 & 21 & -0.71 & +0.31 & -0.54 & +0.08 & -1.09 & -0.09 & -0.97 & +0.19 \\
\hline $\mathrm{Ca}$ II & 5.48 & & 1 & -0.88 & +0.09 & - & - & - & - & - & - \\
\hline Sc II & 2.08 & 0.12 & 13 & -1.09 & -0.12 & -0.86 & -0.24 & -0.75 & -0.40 & -1.37 & -0.21 \\
\hline $\mathrm{Ti} \mathrm{I}$ & 4.20 & 0.07 & 56 & -0.82 & +0.20 & -0.48 & +0.14 & -1.20 & -0.20 & -1.19 & -0.03 \\
\hline Ti II & 4.22 & 0.14 & 8 & -0.80 & +0.17 & - & - & -0.51 & -0.16 & -1.16 & 0.00 \\
\hline V I & 3.11 & 0.09 & 23 & -0.89 & +0.13 & -0.56 & +0.06 & -1.24 & -0.24 & -1.24 & -0.08 \\
\hline V II & 3.01 & & 1 & -0.99 & -0.02 & - & - & - & - & - & - \\
\hline $\mathrm{Cr} \mathrm{I}$ & 4.59 & 0.12 & 27 & -1.08 & -0.06 & -0.82 & -0.20 & -1.19 & -0.19 & -1.08 & +0.08 \\
\hline Cr II & 4.85 & 0.08 & 6 & -0.82 & +0.15 & - & - & - & - & - & - \\
\hline Mn I & 4.24 & 0.10 & 11 & -1.15 & -0.13 & -1.00 & -0.38 & - & - & - & - \\
\hline $\mathrm{Fe} \mathrm{I}$ & 6.48 & 0.07 & 180 & -1.02 & - & -0.62 & - & -1.00 & - & -1.16 & - \\
\hline $\mathrm{Fe}$ II & 6.53 & 0.07 & 17 & -0.97 & - & - & - & -0.35 & - & -1.16 & - \\
\hline Co I & 4.11 & 0.12 & 21 & -0.81 & +0.21 & -0.70 & -0.08 & -0.84 & +0.16 & -1.10 & +0.06 \\
\hline Ni I & 5.15 & 0.11 & 54 & -1.10 & -0.08 & -0.62 & 0.00 & -0.84 & +0.16 & -1.12 & +0.04 \\
\hline $\mathrm{Cu} \mathrm{I}$ & 3.31 & 0.17 & 3 & -0.90 & +0.12 & - & - & -0.64 & +0.36 & -0.80 & +0.36 \\
\hline $\mathrm{Zn} \mathrm{I}$ & 3.78 & & 1 & -0.82 & +0.20 & - & - & -0.39 & +0.61 & -0.92 & +0.24 \\
\hline Y II & 1.21 & 0.12 & 6 & -1.03 & -0.06 & -0.73 & -0.11 & -0.62 & -0.27 & -1.14 & +0.02 \\
\hline $\mathrm{Zr} \mathrm{I}$ & & & & & & & & -1.43 & -0.43 & -1.43 & -0.27 \\
\hline Zr II & 1.91 & 0.13 & 3 & -0.69 & +0.28 & -0.37 & +0.25 & - & - & - & - \\
\hline Ba II & 0.97 & 0.05 & 3 & -1.16 & -0.19 & -0.66 & -0.04 & - & - & - & - \\
\hline La II & 0.24 & 0.25 & 3 & -0.93 & +0.04 & -0.52 & +0.10 & - & - & - & - \\
\hline $\mathrm{Ce}$ II & 0.26 & 0.16 & 5 & -1.32 & -0.35 & -0.65 & -0.03 & -0.68 & -0.33 & -1.21 & -0.05 \\
\hline Pr II & -0.20 & 0.13 & 2 & -0.91 & +0.06 & - & - & - & - & - & - \\
\hline Nd II & 0.56 & 0.13 & 8 & -0.94 & +0.03 & - & - & -0.61 & -0.26 & -1.15 & +0.01 \\
\hline $\mathrm{Eu}$ II & 0.17 & & 1 & -0.34 & +0.63 & - & - & -0.40 & -0.05 & -1.01 & +0.15 \\
\hline
\end{tabular}

that HD 139409 is less luminous than predicted by Mennessier et al. (1997). The derived iron abundance is $[\mathrm{Fe} / \mathrm{H}]=-0.42$ [adopting $\log (\epsilon(\mathrm{Fe}))=7.50]$.

The basic conclusion from the set of abundances listed in Table 4 and displayed in Fig. 7 is that HD 139409 appears to be a mild barium star ${ }^{1}$. The s-process overabundances observed in HD 139409, although quite moderate, are not much smaller than those observed in a yellow symbiotic star like $\mathrm{BD}-21^{\circ} 3873$, which exhibit overabundances of s-process

\footnotetext{
${ }^{1}$ Interestingly, Pinsonneault et al. (1984) quoting a private communication from E. Luck, remark that Luck "is completing an abundance analysis of HD 139409, which will show that the light s-process elements do have $(\sim+1.5 \mathrm{dex})$ enhancements, while heavier s-process element enhancements are much smaller $(\sim+0.3$ dex $)$ ". Luck's study seems to have never been published.
}

elements in the range 0.3-0.8 dex (Smith et al. 1997). With $[\mathrm{Fe} / \mathrm{H}]=-0.4$, HD 139409 is, however, not as metal-deficient as the other stars considered in this paper.

\section{Statistics of binarity among metal-deficient Ba stars}

The list of confirmed or suspected metal-deficient barium stars remaining after the screening process based on abundances (as described in Sect. 3) is given in Table 5. The possible binary nature of those stars may be assessed using three different methods:

\footnotetext{
- checking for radial-velocity variations;

- checking for astrometric orbital motion, directly from the Hipparcos astrometric data;
} 
Table 4. Abundances for HD 139409, in the scale where $\log \epsilon(\mathrm{H})=$ 12 , and normalized with respect to the solar abundances (Grevesse \& Sauval 1998). The standard deviations $\sigma$ and the number $N$ of lines used in the analysis are also given.

\begin{tabular}{lrrrrr}
\hline \hline Element $(X)$ & $Z$ & $\log \epsilon(X)$ & $\sigma$ & $N$ & {$[X / \mathrm{Fe}]$} \\
\hline C I & 6 & 8.46 & 0.10 & 4 & +0.36 \\
$\mathrm{Na}$ I & 11 & 6.02 & 0.05 & 5 & +0.11 \\
$\mathrm{Mg}$ I & 12 & 7.31 & 0.12 & 6 & +0.15 \\
$\mathrm{Si} \mathrm{I}$ & 14 & 7.33 & 0.06 & 20 & +0.20 \\
$\mathrm{Ca}$ I & 20 & 6.20 & 0.07 & 6 & +0.26 \\
$\mathrm{Sc}$ II & 21 & 2.79 & 0.06 & 10 & +0.04 \\
Ti I & 22 & 4.77 & 0.09 & 51 & +0.17 \\
Ti II & 22 & 4.72 & 0.11 & 4 & +0.12 \\
V I & 23 & 3.76 & 0.11 & 23 & +0.18 \\
Cr I & 24 & 5.21 & 0.11 & 11 & -0.04 \\
Cr II & 24 & 5.36 & 0.09 & 3 & +0.11 \\
Mn I & 25 & 5.13 & 0.11 & 9 & +0.16 \\
Fe I & 26 & 7.08 & 0.08 & 136 & - \\
Fe II & 26 & 7.08 & 0.12 & 14 & - \\
Ni I & 28 & 5.86 & 0.07 & 44 & +0.03 \\
Y I & 39 & 2.22 & 0.10 & 2 & +0.40 \\
Y II & 39 & 2.41 & 0.07 & 6 & +0.59 \\
Zr I & 40 & 2.58 & 0.09 & 3 & +0.40 \\
Zr II & 40 & 2.78 & 0.12 & 3 & +0.60 \\
Ba II & 56 & 1.89 & 0.12 & 3 & +0.18 \\
La II & 57 & 1.05 & 0.12 & 3 & +0.30 \\
Ce II & 58 & 1.46 & 0.14 & 5 & +0.30 \\
Nd II & 60 & 1.38 & 0.09 & 6 & +0.30 \\
\hline & & & & &
\end{tabular}

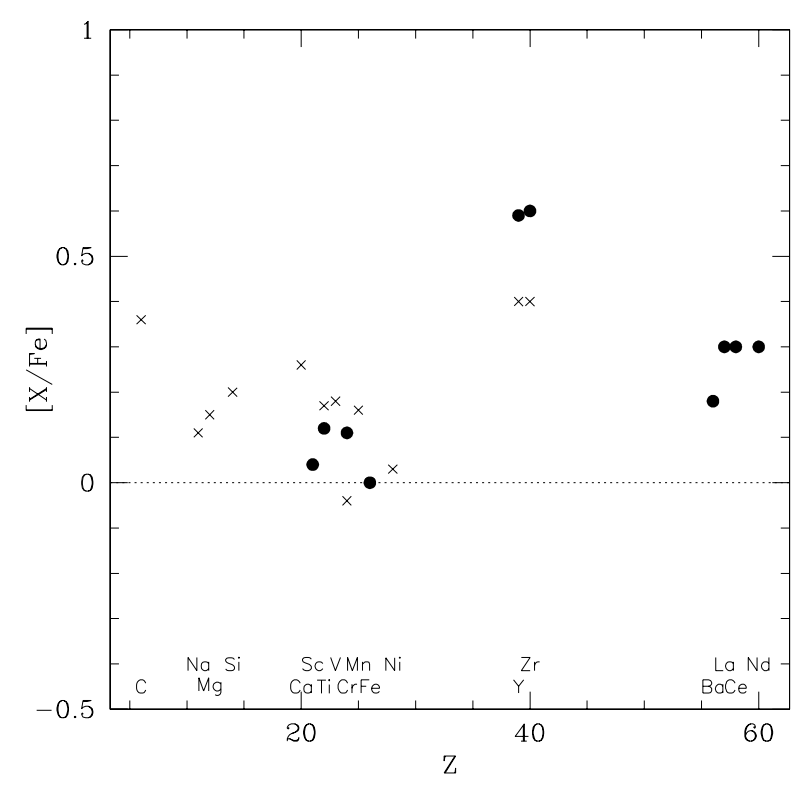

Fig. 7. Abundance pattern in HD 139409 (abundances derived from ionized species are represented by black dots, and from neutral species by crosses).

- checking for astrometric orbital motion, indirectly from a comparison of the Hipparcos and Tycho-2 proper motions.

These methods are now described in turn.

\subsection{Radial-velocity variations}

Several stars from Table 5 (namely HIP 4347, HIP 29740, HIP 34795, HIP 55852, HIP 58956, HIP 76605 and $\mathrm{BD}+3^{\circ} 2688$ ) have been monitored for many years using the CORAVEL spectrovelocimeter (Baranne et al. 1979), as part of a larger program aiming at finding the frequency of spectroscopic binaries among s-process-rich late-type giants (see Jorissen \& Mayor 1988, 1992; Jorissen et al. 1998, for details and other results from this CORAVEL monitoring). Individual radial-velocity measurements for those stars (in the CORAVEL-ELODIE system as defined in Udry et al. 1999) are given in Table 7 or in Udry et al. (1998). For a few other stars (HIP 43042, CS 22942-019 and CS 22948-027), radial velocities were monitored using other instruments, and their results were taken from the literature (Preston \& Sneden 2001; Začs et al. 2005).

Orbits were already available for HIP 4347 and HIP 29740 (Udry et al. 1998), HIP 43042 (Začs et al. 2005), as well as for CS 22942-019 and CS 22948-027 (Preston \& Sneden 2001). A new orbital solution has been derived for $\mathrm{BD}+04^{\circ} 2466$ (Table 6 and Fig. 8). The binary nature of those stars is therefore beyond doubt.

The radial-velocity standard deviation of HIP 58596 is larger than expected based on the uncertainty on one measurement (Fig. 9), but no satisfactory orbital solution could be found. A 3-d orbit (with an eccentricity of 0.30 ) is possible, but this short orbital period is not consistent with the giant nature of HIP 58596, which imposes orbital periods of at least $20 \mathrm{~d}$ (see Fig. 4 of Pourbaix et al. 2004). The large standard deviation exhibited by HIP 58596 is therefore very likely another example of the large intrinsic jitter often observed for metal-deficient stars, as discussed by McClure (1984) and Carney et al. (2003).

A 14 y radial-velocity monitoring for HIP 34795 with the northern and southern CORAVELs is not very conclusive either, mostly because there are difficulties in finding the zeropoint offset between the two instruments for such large radial velocities (Udry et al. 1999). When a $-1 \mathrm{~km} \mathrm{~s}^{-1}$ offset is applied to the northern velocities (with respect to the values listed in Table 7), a long-term trend seems to be present, albeit with some superimposed jitter (Fig. 11). The analysis of the Hipparcos astrometric data presented in Sect. 4.2 suggests that the star might be binary, although the evidence is not very conclusive.

Finally, there is no sign of radial-velocity variations for $\mathrm{BD}+3^{\circ} 2688$ (Fig. 10).

\subsection{Orbital motion from Hipparcos astrometric data}

A tailored reprocessing of the Hipparcos Intermediate Astrometric Data (hereafter IAD; van Leeuwen \& Evans 1998) makes it possible to look for a possible orbital signature in the astrometric motion, following the method outlined by Pourbaix \& Jorissen (2000), Pourbaix \& Boffin (2003), Pourbaix (2004) and applied to barium stars by Jorissen et al. (2004b). We give here only a brief summary of the method.

The basic idea is to quantify the likelihood of the fit of the Hipparcos astrometric data with an orbital model. For that 
Table 5. Binary properties of confirmed or suspected metal-deficient barium stars. The column labeled "Ba" indicates whether detailed chemical analyses have confirmed the Ba nature of the star. The columns labeled $\chi_{\mu_{\mathrm{HIP}}-\mu_{\mathrm{Tyc}}}^{2}$ and $\operatorname{Prob}\left(\chi^{2}>\chi_{\mu_{\mathrm{HIP}}-\mu_{\mathrm{Tyc}}}^{2}\right)$ provide the $\chi^{2}$ (and its associated probability) involved in the comparison of Hipparcos and Tycho-2 proper motions (see text for details). The column "Bin." has been set to "y" if the first kind risk of rejecting the null hypothesis that the Tycho and Hipparcos proper motions are equal is smaller than 10\%. The column labeled IAD indicates whether the signature of an orbital motion is present in the Hipparcos Intermediate Astrometric Data, according to the various tests described in the text. The column labeled $\sigma(V r)$ provides the radial-velocity standard deviation, $\Delta t$ and $N$ correspond to the time span and number of observations, respectively. The columns labeled $P$ and "Ref" list the orbital period (when available) and the reference for the radial velocity and/or orbital data. The column "Binary" gives the final binarity diagnostics.

\begin{tabular}{|c|c|c|c|c|c|c|c|c|c|c|c|c|c|c|}
\hline \multirow[t]{2}{*}{ "HIP } & \multirow[t]{2}{*}{$\overline{\mathrm{HD} / \mathrm{DM}}$} & \multirow[t]{2}{*}{$\mathrm{Ba}$} & \multirow[t]{2}{*}{ Ref. } & \multirow[t]{2}{*}{$\bar{\tau} \varpi$ (mas) } & \multicolumn{3}{|c|}{$\mu_{\mathrm{HIP}}-\mu_{\mathrm{Tyc}}$} & \multirow[t]{2}{*}{ IAD } & \multirow{2}{*}{$\begin{array}{l}\sigma(V r) \\
\left(\mathrm{km} \mathrm{s}^{-1}\right)\end{array}$} & \multirow{2}{*}{$\begin{array}{l}\Delta t \\
\text { (d) }\end{array}$} & \multirow[t]{2}{*}{$\bar{N}$} & \multirow{2}{*}{$\begin{array}{l}P \\
\text { (d) }\end{array}$} & \multirow[t]{2}{*}{ Ref. } & \multirow[t]{2}{*}{ Binary } \\
\hline & & & & & $\chi_{\mathrm{obs}}^{2}$ & $\operatorname{Prob}\left(\chi^{2}>\chi_{\mathrm{obs}}^{2}\right)$ & Bin. & & & & & & & \\
\hline 4347 & 5424 & & & $0.22 \pm 1.42$ & 1.05 & 0.59 & $\mathrm{n}$ & $\mathrm{n}$ & 2.32 & 3306 & 13 & 1881 & 6 & $\mathrm{y}$ \\
\hline 11595 & 15589 & & & $2.03 \pm 1.21$ & 1.63 & 0.44 & $\mathrm{n}$ & $\mathrm{n}$ & - & - & - & - & & $?$ \\
\hline 25161 & $-27^{\circ} 2233$ & & & $0.89 \pm 1.35$ & 0.23 & 0.89 & $\mathrm{n}$ & $\mathrm{n}$ & - & - & - & - & & $?$ \\
\hline 29740 & 43389 & & & $-1.25 \pm 1.00$ & 4.54 & 0.10 & $\mathrm{y}$ & $\mathrm{y}$ & 3.85 & 3350 & 24 & 1689 & 6 & $\mathrm{y}$ \\
\hline 34795 & 55496 & $\mathrm{y}$ & 5 & $2.44 \pm 1.04$ & 0.02 & 0.99 & $\mathrm{n}$ & $\mathrm{y}$ & 0.73 & 5121 & 24 & - & 2 & $\mathrm{y} ?$ \\
\hline 58596 & 104340 & $\mathrm{y}$ & 3 & $-0.97 \pm 1.09$ & 3.39 & 0.18 & $\mathrm{n}$ & $\mathrm{n}$ & 1.64 & 2587 & 16 & - & 2 & $\mathrm{n}$ \\
\hline 69834 & 123396 & & & $1.73 \pm 0.86$ & 1.98 & 0.27 & $\mathrm{n}$ & $\mathrm{n}$ & - & - & - & - & & $?$ \\
\hline 76605 & 139409 & $\mathrm{y}$ & 2 & $5.51 \pm 1.14$ & 4.98 & 0.08 & $\mathrm{y}$ & $\mathrm{n}$ & 0.66 & 1478 & 2 & - & 2 & $\mathrm{y} ?$ \\
\hline 97874 & 187762 & & & $2.07 \pm 1.53$ & 9.00 & 0.01 & $\mathrm{y}$ & $\mathrm{y}$ & - & - & - & - & & $\mathrm{y}$ \\
\hline 107478 & 206983 & $\mathrm{y}$ & 3 & $3.75 \pm 1.86$ & 2.94 & 0.23 & $\mathrm{n}$ & $\mathrm{y}$ & - & - & - & - & & $\mathrm{y} ?$ \\
\hline \multicolumn{15}{|c|}{ Additional stars (from refs. (1), (5) and (10)) } \\
\hline 43042 & $+75^{\circ} 348$ & $\mathrm{y}$ & 1 & $1.02 \pm 1.32$ & 0.12 & 0.94 & $\mathrm{n}$ & $\mathrm{y}$ & 4.64 & 1436 & 33 & 1042 & 11 & $\mathrm{y}$ \\
\hline \multirow[t]{4}{*}{55852} & $+4^{\circ} 2466$ & $\mathrm{y}$ & 4,5 & $0.96 \pm 1.83$ & 0.43 & 0.81 & $\mathrm{n}$ & $\mathrm{n}$ & 3.38 & 6191 & 41 & 4592 & 2 & $\mathrm{y}$ \\
\hline & $+3^{\circ} 2688$ & $\mathrm{y}$ & 5 & - & - & - & - & - & 0.45 & 986 & 7 & - & 2 & $\mathrm{n}$ ? \\
\hline & CS 22942-019 & $\mathrm{y}$ & 10 & - & - & - & - & - & 3.42 & 3274 & 15 & 2800 & 10 & $\mathrm{y}$ \\
\hline & CS 22948-027 & $\mathrm{y}$ & 10 & - & - & - & - & - & 1.87 & 2560 & 13 & 505 & 10 & $\mathrm{y}$ \\
\hline
\end{tabular}

References to the table: (1) Začs et al. (2000); (2) this work (3) Junqueira \& Pereira (2001); (4) Burris et al. (2000); (5) Luck \& Bond (1991); (6) Udry et al. (1998); (10) Preston \& Sneden (2001); (11) Začs et al. (2005).

Table 6. Orbital elements for $\mathrm{BD}+04^{\circ} 2466$.

\begin{tabular}{ll}
\hline \hline$P(\mathrm{~d})$ & $4592.7 \pm 51.1$ \\
$e$ & $0.286 \pm 0.02$ \\
$\omega(\mathrm{deg})$ & $266.8 \pm 5.3$ \\
$V_{\gamma}\left(\mathrm{km} \mathrm{s}^{-1}\right)$ & $+39.29 \pm 0.11$ \\
$K\left(\mathrm{~km} \mathrm{~s}^{-1}\right)$ & $5.67 \pm 0.12$ \\
$T(\mathrm{JD})$ & $2445076.6 \pm 73.0$ \\
$f(M)\left(M_{\odot}\right)$ & 0.076 \\
$a_{1} \sin i(\mathrm{Gm})$ & 343.18 \\
$N$ & 41 \\
\hline
\end{tabular}

purpose, Pourbaix \& Arenou (2001) (see also Jancart et al. 2005 ) introduced several statistical indicators which allow us to decide whether to keep or to discard an orbital solution. Those indicators relevant to our purpose are the following:

- The addition of 4 supplementary parameters (the four Thiele-Innes orbital constants) describing the orbital motion should result in a statistically significant decrease of the $\chi^{2}$ for the fit of the $N$ IAD with an orbital model with 9 free parameters $\left(\chi_{T}^{2}\right)$, as compared to a fit with a single-star solution with 5 free parameters $\left(\chi_{S}^{2}\right)$. This criterion is expressed by an $F$-test:

$$
\operatorname{Pr}_{2}=\operatorname{Pr}[\hat{F}<F(4, N-9)] \text {, }
$$

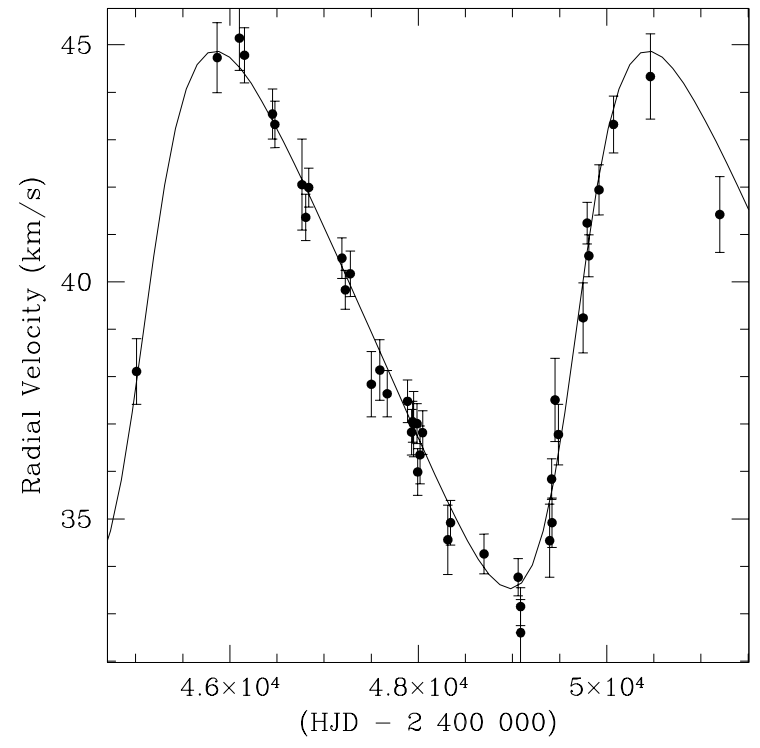

Fig. 8. Radial velocities as a function of heliocentric Julian Day for $\mathrm{BD}+4^{\circ} 2466$, superimposed on the orbital solution corresponding to the orbital elements listed in Table 6.

where

$\hat{F}=\frac{N-9}{4} \frac{\chi_{\mathrm{S}}^{2}-\chi_{\mathrm{T}}^{2}}{\chi_{\mathrm{T}}^{2}}$. 


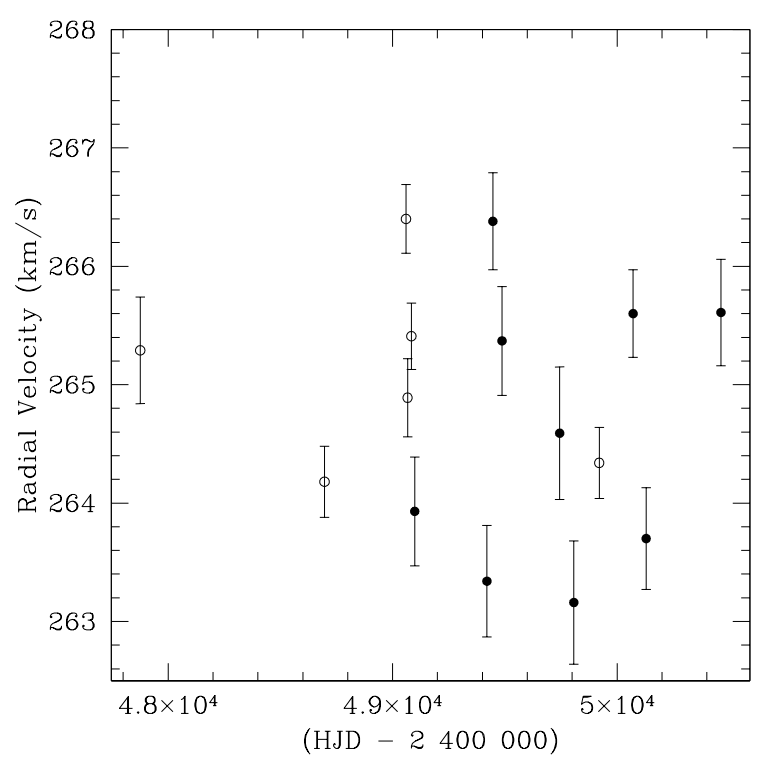

Fig. 9. Radial velocities as a function of heliocentric Julian Day for HIP 58596 = HD 104340 (open circles correspond to measurements obtained with the southern CORAVEL, and filled circles with the northern one).

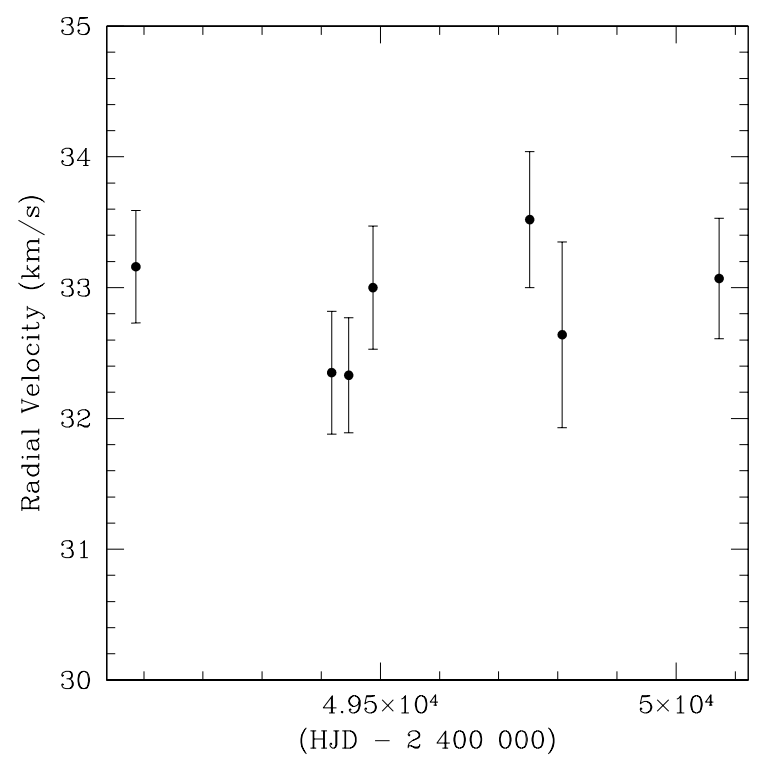

Fig. 10. Radial velocities as a function of heliocentric Julian Day for $\mathrm{BD}+3^{\circ} 2688$.

$\mathrm{Pr}_{2}$ is thus the first kind risk associated with the rejection of the null hypothesis: "there is no orbital wobble present in the data".

- Getting a substantial reduction of the $\chi^{2}$ with the ThieleInnes model does not necessarily imply that the four Thiele-Innes constants $A, B, F, G$ are significantly different from 0 . The first kind risk associated with the rejection of the null hypothesis "the orbital semi-major axis is equal to zero" may be expressed as

$\operatorname{Pr}_{3}=\operatorname{Pr}\left[\chi_{A B F G}^{2}<\chi^{2}(4)\right]$,

where

$\chi_{A B F G}^{2}=\boldsymbol{X}^{t} \boldsymbol{V}^{-1} \boldsymbol{X}$,

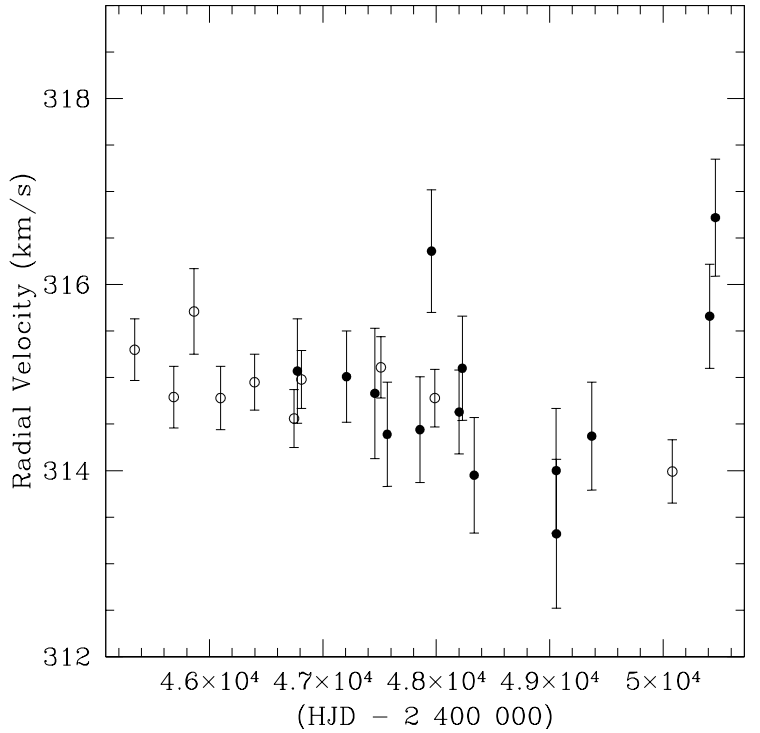

Fig. 11. Radial velocities as a function of heliocentric Julian Day for HIP $34795=$ HD 55496. The symbols are as in Fig. 9. With respect to the data listed in Table 7, an offset of $-1 \mathrm{~km} \mathrm{~s}^{-1}$ has been applied to the northern measurements to make them consistent with the southern ones.

and $\boldsymbol{X}$ is the vector of components $A, B, F, G$ and $\boldsymbol{V}$ is its covariance matrix ${ }^{2}$.

- For the orbital solution to be a significant one, its parameters should not be strongly correlated with the other astrometric parameters (e.g., the proper motion). In other words, the covariance matrix of the astrometric solution should be dominated by its diagonal terms, as measured by the efficiency $\epsilon$ of the matrix being close to 1 (Eichhorn 1989). The efficiency is simply expressed by

$\epsilon=\sqrt[m]{\frac{\Pi_{k=1}^{m} \lambda_{k}}{\prod_{k=1}^{m} \boldsymbol{V}_{k k}}}$,

where $\lambda_{k}$ and $\boldsymbol{V}_{k k}$ are respectively the eigenvalues and the diagonal terms of the covariance matrix $\boldsymbol{V}$.

With the above notations, the requirements for a star to qualify as a binary is then

$\alpha \equiv\left(\operatorname{Pr}_{2}+P r_{3}\right) / \epsilon \leq 0.02$,

where the threshold value of 0.02 has been chosen to minimize false detections (Jorissen et al. 2004b).

Hipparcos data are, however, seldom precise enough to derive the orbital elements from scratch. Therefore, when a spectroscopic orbit is available beforehand, it is advantageous to import $e, P, T$ from the spectroscopic orbit and to derive the remaining astrometric elements (as done by Pourbaix \& Jorissen 2000; Pourbaix \& Boffin 2003). If a spectroscopic orbit is not

\footnotetext{
${ }^{2}$ Since it may be shown that $\chi_{\mathrm{S}}^{2}-\chi_{\mathrm{T}}^{2}=\chi_{A B F G}^{2}$, the $\mathrm{Pr}_{2}$ and $P r_{3}$ tests are in fact equivalent provided that $\chi_{\mathrm{T}}^{2} \sim N-9$. Thus, if $\mathrm{Pr}_{2}$ and $\mathrm{Pr}_{3}$ are significantly different, it means either that the Thiele-Innes orbital model does not fit the data very well $\left(\chi_{\mathrm{T}}^{2} \gg N-9\right)$, or that it fits much better than could be expected $\left(\chi_{\mathrm{T}}^{2} \ll N-9\right)$. We are indebted to $\mathrm{L}$. Lindegren for this clarification (see also Jancart et al. 2005).
} 

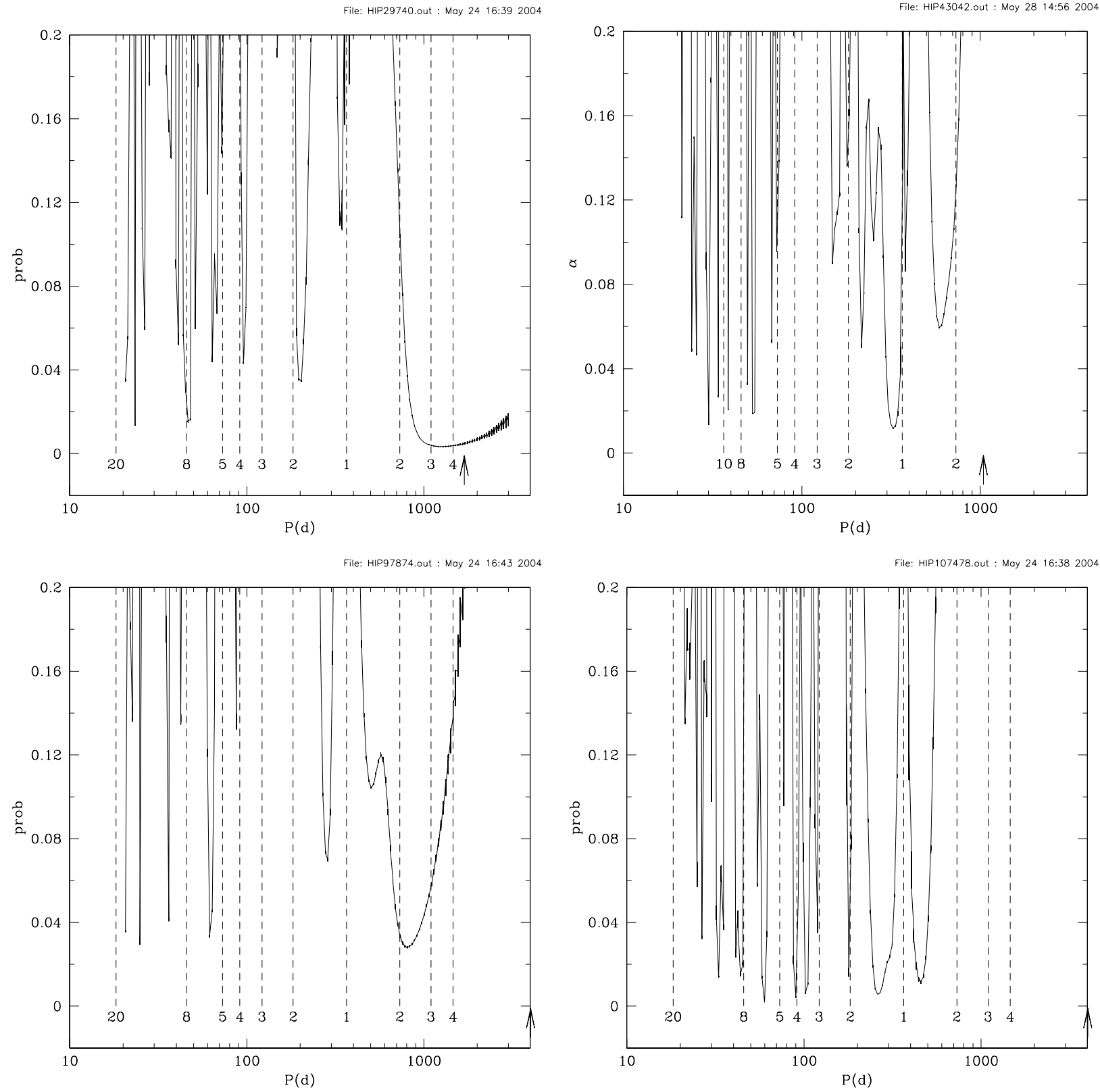

Fig. 12. The $\alpha$ statistics (Eq. (6)) as a function of the trial orbital period (assuming $e=0$ ) for metal-deficient barium stars. The period from the spectroscopic orbit (represented by an arrow) of HIP 29740 (upper left panel) indeed lies within the range of minimum $\alpha$ values. By comparison, the suspected binaries HIP 97874 and HIP 107478 are likely to have periods $P \sim 800 \mathrm{~d}$ and $\sim 250-450 \mathrm{~d}$, respectively. For comparison, stars non-flagged as binaries have no $(e, P, T)$ grid points with $\alpha<0.02$ (see Fig. 13). The vertical dashed lines represent multiple, or integer fractions, of $1 \mathrm{y}$. At those periods, there is a strong correlation between the parallactic and orbital signals, which degrades the $\alpha$ statistics and makes binaries difficult to find at those $1-\mathrm{y}$ alias periods.

available, trial $(e, P, T)$ triplets scanning a regular grid (with $10 \leq P(\mathrm{~d}) \leq 5000$ imposed by the Hipparcos scanning law and the mission duration) may be used. The quality factor $\alpha$ is then computed for each trial $(e, P, T)$ triplet, and if there exist triplets yielding $\alpha<0.02$, the star is flagged as a binary. To test its success rate, this method has been applied by Jorissen et al. (2004b) on a sample of barium stars. These authors show that, when $\varpi>5$ mas and $100<P(\mathrm{~d})<4000$, the (astrometric) binary detection rate is close to $100 \%$, i.e., the astrometric method recovers all known spectroscopic binaries (see also Jancart et al. 2005). When the orbit is not known beforehand, the method makes it even possible to find a good estimate for the orbital period, provided, however, that the true period is not an integer fraction, or a multiple, of one year. Here the method is applied to the sample of metal-deficient barium stars listed in Table 5.

The method flags as definite binaries the stars HIP 29740, 34795, 43042, 97874 and 107478 (Figs. 12 and 13). In two cases (HIP 29740 and 43042), the IAD method thus confirms the conclusion from the radial-velocity monitoring, but yields as well three new binaries (HIP 34795, 97874 and 107478). Two spectroscopic binaries (HIP 4347 and 

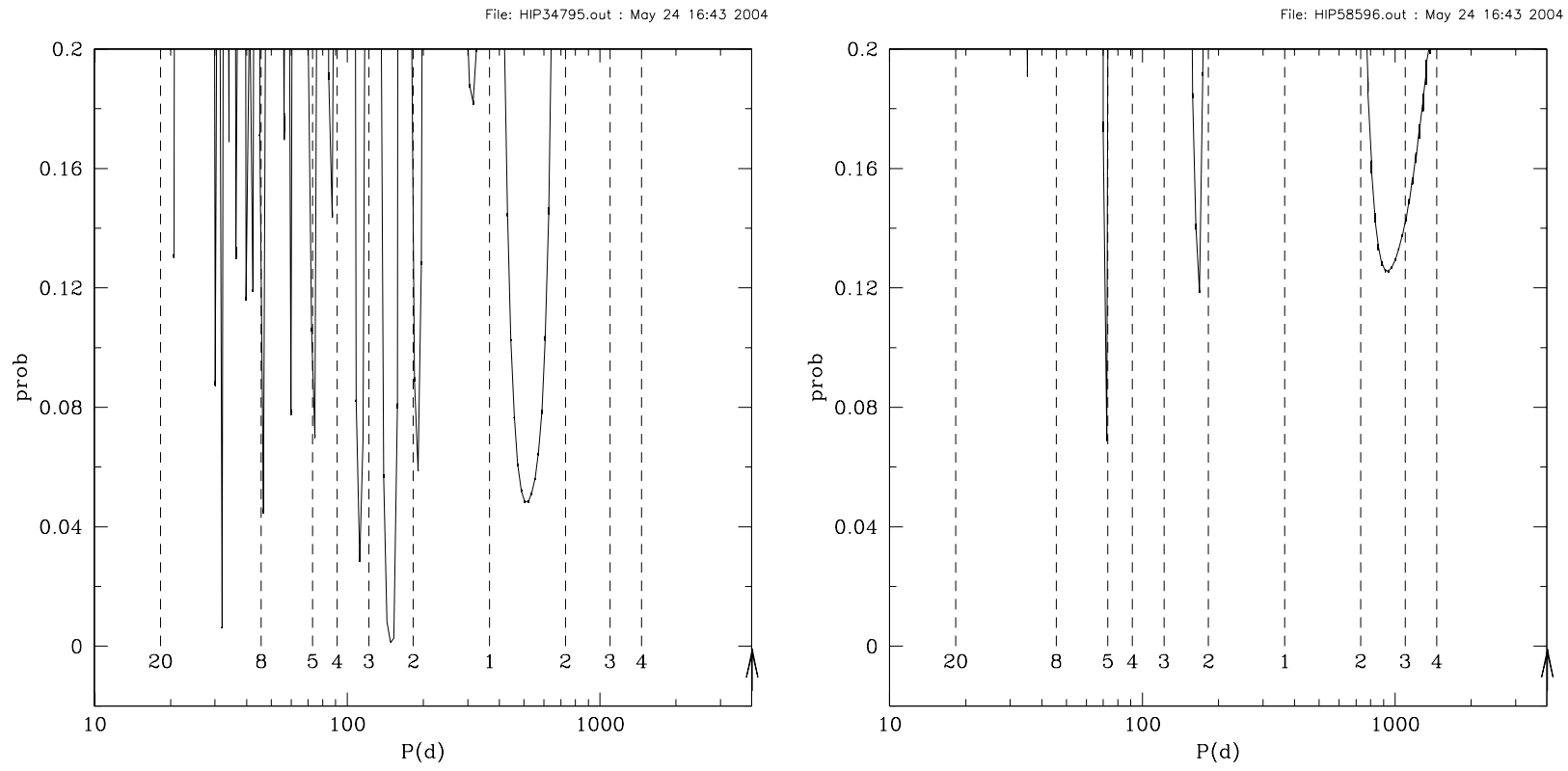

Fig. 13. Same as Fig. 12 for HIP 34795 (left panel, flagged as binary) and HIP 58596 (right panel, non-binary).

HIP 55852) are not detected by the IAD method because of their small parallax or long orbital period. The non-binary nature of HIP 58596, already suspected from the radial-velocity data, is confirmed by the analysis of the IAD (Fig. 13).

\subsection{Orbital motion from a comparison of Hipparcos and Tycho-2 proper motions}

Kaplan \& Makarov (2003) suggested that the comparison of Hipparcos and Tycho-2 (Høg et al. 2000b) proper motions offers a way to detect binaries with long periods (typically from 2000 to 4000 d). The Hipparcos proper motion, being based on observations spanning only $3 \mathrm{y}$, may be altered by the orbital motion, especially for systems with periods in the range of 2000 to $4000 \mathrm{~d}$ whose orbital motion was not recognized by Hipparcos. On the other hand, this effect should average out in the Tycho-2 proper motion, which is derived from observations covering a much longer time span. This method, already used by Makarov (2004), Pourbaix (2004) and Jancart et al. (2006), works best when applied to stars with parallaxes in excess of about 5 mas.

The method evaluates the quantity

$\chi_{\mathrm{obs}}^{2}=\left(\boldsymbol{\mu}_{\mathrm{HIP}}-\boldsymbol{\mu}_{\mathrm{Tyc}}\right)^{t} \boldsymbol{W}^{-1}\left(\boldsymbol{\mu}_{\mathrm{HIP}}-\boldsymbol{\mu}_{\mathrm{Tyc}}\right)$,

where $\boldsymbol{\mu}_{\mathrm{HIP}}$ and $\boldsymbol{\mu}_{\mathrm{Tyc}}$ are the vectors of $\alpha$ and $\delta$ components of the Hipparcos and Tycho- 2 proper motions, respectively, and $\boldsymbol{W}$ is the associated $2 \times 2$ variance-covariance matrix. The covariance between $\mu_{\alpha, \mathrm{HIP}}$ and $\mu_{\delta, \mathrm{HIP}}$, as provided by field $\mathrm{H} 28$ of the Hipparcos catalogue (ESA 1997) and the correlation between Tycho-2 and Hipparcos proper motions, as encapsulated in the quantity $R$ of Table 1 of Høg et al. (2000a), have both been considered (see Jancart et al. 2006, for details).

Since the above quantity follows a $\chi^{2}$ probability distribution function with 2 degrees of freedom, it is then possible to compute the probability $\operatorname{Prob}\left(\chi^{2}>\chi_{\mathrm{obs}}^{2}\right)$, giving the first kind risk of rejecting the null hypothesis $\boldsymbol{\mu}_{\mathrm{Tycho}}=\boldsymbol{\mu}_{\mathrm{HIP}}$ while it is actually true. This probability is listed in Table 5, along with $\chi_{\text {obs }}^{2}$, and the star is flagged as binary if Prob $<0.1$.

Only HIP 29740, HIP 76605 and HIP 97874 satisfy the test at the $10 \%$ threshold. Note, however, that all the other stars have parallaxes smaller than 5 mas, which make the test less efficient.

\section{Summary of the binary criteria and discussion}

The situation may be summarized as follows (see also last column of Table 5):

- Definite binaries with known orbits: HIP 4347, HIP 29740 (passes all three binarity tests), HIP 43042, HIP 55852, CS 22942-019, CS 22948-027;

- Suspected binaries from astrometric data (either IAD or proper motions; no or inconclusive radial-velocity data): HIP 34795, HIP 76605, HIP 97874 (both astrometric tests yield positive results), HIP 107478;

- Data inconclusive (mainly because of too small a parallax): HIP 11595, HIP 25161, HIP 69834;

- Non-binary stars (mainly from radial-velocity data, not contradicted by astrometry): HIP $58596, \mathrm{BD}+3^{\circ} 2688$.

The latter two non-binary stars are in fact good candidate thermally-pulsing AGB stars, as revealed by their location in the HR diagram (Table 2 and right panel of Fig. 1). Hence, they must not be binaries.

Finally, we come to the central question of this paper: why do the metal-deficient barium stars, despite being binaries and occupying the same location of the HR diagram as YSyS, do not exhibit symbiotic activity? Three possible answers have been suggested in this paper:

(i) Some among the stars listed in Table 2 and displayed in Fig. 1 are in fact not barium stars (especially HIP $80843=$ HD 148897). 


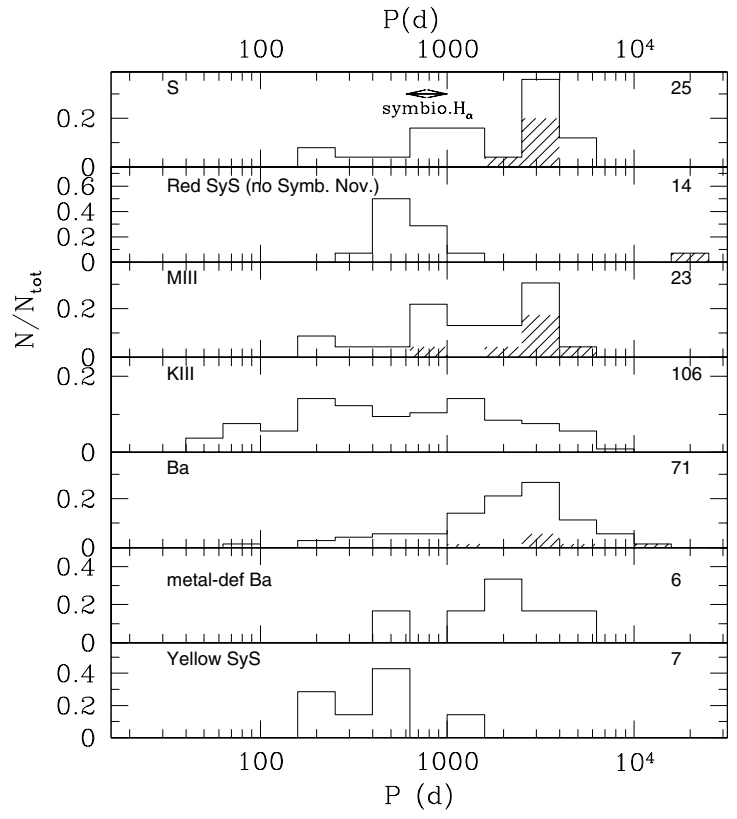

Fig. 14. Comparison of the period distributions for samples of binary systems with different kinds of red giant primaries: S stars (Jorissen et al. 1998), red SyS (excluding symbiotic novae and symbiotic Miras; Mürset \& Schmid 1999), M giants (Jorissen et al. 2004a) and K giants (Mermilliod 1996). The lower two panels present the orbital-period distribution for barium stars (Jorissen et al. 1998) and yellow SyS (Mürset \& Schmid 1999). In the S star panel, the arrow marked $\mathrm{H}_{\alpha}$ indicates the period range where binary $\mathrm{S}$ stars exhibit $\mathrm{H}_{\alpha}$ emission as a signature of weak symbiotic activity. The shaded area marks stars with only a lower limit available on their orbital period. The numbers in the upper right corner of each panel correspond to the sample size.

(ii) Among those which are barium stars, some seem to lie on the TP-AGB, and thus need not be binaries (HIP $58596=$ HD 104340, BD $\left.+3^{\circ} 2688\right)$. They therefore cannot exhibit symbiotic activity.

(iii) Finally, there remain a few genuine metal-deficient barium stars in the sample. Why are they not symbiotic stars? It seems that the answer to that question lies in the different period distributions for YSyS and metal-deficient barium stars: YSyS have shorter orbital period than metaldeficient barium stars, as seen in Fig. 14. This argument seems to apply especially to HIP 29740 (=HD 43389), which has been assigned a very bright absolute visual magnitude of -3.5 by the maximum likelihood method of Mennessier et al. (1997). It is therefore expected to have a rather strong mass loss rate, and be a good candidate YSyS. However, with its orbital period of 1689 d, it lies at the long-period edge of the period distribution of YSyS (Fig. 14). The same difference seems to exist between the period distributions of red symbiotics and binary S stars (Van Eck \& Jorissen 2002, and Fig. 14).

However, a firm conclusion on this issue should await the determination of the orbital periods for the metal-deficient barium stars flagged as binaries by the IAD method (especially HIP 11595, HIP 25161, HIP 69834, HIP 97874, HIP 107478), so as to make the comparison between the orbital period distributions of metal-deficient barium stars and YSyS more meaningful.

Acknowledgements. This work was performed in the framework of the NATO Collaborative Linkage Grant SA (PST.CLG.979128)6774/FP. We thank D. Pourbaix for the processing of the Hipparcos data of the metal-deficient stars. F. Carrier and X. Bonfils are thanked for obtaining the Arcturus and HD 139409 spectra. L.Z. thanks I. Platais for valuable discussions and support. The Fonds National Suisse de la Recherche Scientifique has funded the operations of the CORAVEL spectrometer and of the Swiss 1-m telescope installed at the Haute-Provence Observatory.

\section{References}

Allen, D. A. 1982, in The Nature of Symbiotic Stars, ed. M. Friedjung, \& R. Viotti (Dordrecht: Reidel), IAU Coll., 70, 27

Allende Prieto, C., García López, R. J., Lambert, D. L., \& Gustafsson, B. 1999, ApJ, 527, 879

Baranne, A., Mayor, M., \& Poncet, J. L. 1979, Vistas Astron., 23, 279

Barbuy, B., Jorissen, A., Rossi, S. C. F., \& Arnould M. 1992, A\&A, 262, 216

Bessell, M. S., Castelli, F., \& Plez, B. 1998, A\&A, 333, 231

Boyle, R. J., \& McClure, R. D. 1975, PASP, 87, 17

Burris, D. L., Pilachowski, C. A., Armandroff, T. E., et al. 2000, ApJ, 544,302

Carney, B. W., Latham, D. W., Stefanik, R. P., Laird, J. B., \& Morse, J. A. 2003, AJ, 125, 293

Clayton, D. D. 1988, MNRAS, 234, 1

Corradi, R., \& Schwarz, H. E. 1997, in Physical Processes in Symbiotic Binaries and Related Systems (Warsaw: Copernicus Foundation for Polish Astronomy), 147

Corradi, R. L. M., Brandi, E., Ferrer, O. E., \& Schwarz, H. E. 1999, A\&A, 343, 841

Drake, S. A., Simon, T., \& Linsky, J. L. 1987, AJ, 93, 163

Eichhorn, H. 1989, Bull. Astron. Inst. Czechosl., 40, 394

ESA 1997, The Hipparcos and Tycho Catalogues, ESA

Galazutdinov, G. 1992, Special Astrophys. Obs. Prepr., 92, 52

Grauer, A. D., \& Bond, H. E. 1981, PASP, 93, 630

Grevesse, N., \& Sauval, A. J. 1998, Space Sci. Rev., 85, 161

Griffin, R. F., \& Keenan, P. C. 1992, The Observatory, 112, 168

Gustafsson, B., Bell, R. A., Eriksson, K., \& Nordlund, A. 1975, A\&A, 42,407

Hinkle, K., Wallace, L., Valenti, J., \& Harmer, D. 2000, Visible and Near Infrared Atlas of the Arcturus Spectrum 3727-9300 ̊ (San Francisco: ASP)

Høg, E., Fabricius, C., Makarov, V. V., et al. 2000a, A\&A, 357, 367

Høg, E., Fabricius, C., Makarov, V. V., et al. 2000b, A\&A, 355, L27

Jancart, S., Jorissen, A., Babusiaux, C., \& Pourbaix, D. 2005, A\&A, in press

Jancart, S., Jorissen, A., Babusiaux, C., \& Pourbaix, D. 2006, A\&A, in preparation

Jeffries, R. D., \& Stevens, I. R. 1996, MNRAS, 279, 180

Jorissen, A. 2003a, in Symbiotic stars probing stellar evolution, ed. R. L. M. Corradi, J. Mikołajewska, \& T. J. Mahoney, (San Francisco), ASP Conf. Ser., 303, 25

Jorissen, A. 2003b, in Asymptotic Giant Branch Stars, ed. H. Habing, \& H. Olofsson (New York: Springer Verlag), 461

Jorissen, A., Famaey, B., Dedecker, M., et al. 2004a, Rev. Mex. Astron. Astrof. Conf. Ser., 21, 71 
Jorissen, A., Jancart, S., \& Pourbaix, D. 2004b, in Spectroscopically and Spatially Resolving the Components of Close Binary Stars, ed. R. W. Hilditch, H. Hensberge, \& K. Pavlovski (San Francisco), ASP Conf. Ser., 318, 141

Jorissen, A., \& Mayor, M. 1988, A\&A, 198, 187

Jorissen, A., \& Mayor, M. 1992, A\&A, 260, 115

Jorissen, A., Schmitt, J. H. M. M., Carquillat, J. M., Ginestet, N., \& Bickert, K. F. 1996, A\&A, 306, 467

Jorissen, A., Van Eck, S., Mayor, M., \& Udry, S. 1998, A\&A, 332, 877

Junqueira, S., \& Pereira, C. B. 2001, AJ, 122, 360

Kaplan, G. H., \& Makarov, V. V. 2003, Astron. Nachrichten, 324, 419

Keenan, P. C., \& McNeil, R. C. 1989, ApJS, 71, 245

Kyröläinen, J., Tuominen, I., Vilhu, O., \& Virtanen, H. 1986, A\&AS, 65,11

Lattanzio, J. C. 1991, ApJS, 76, 215

Lü, P. K. 1991, AJ, 101, 2229

Lü, P. K., Dawson, D. W., Upgren, A. R., \& Weis, E. W. 1983, ApJS, 52,169

Luck, R. E. 1991, ApJS, 75, 579

Luck, R. E., \& Bond, H. E. 1991, ApJS, 77, 515

MacConnell, D. J., Frye, R. L., \& Upgren, A. R. 1972, AJ, 77, 384

Makarov, V. V. 2004, ApJ, 600, L71

Mayor, M., Pepe, F., Queloz, D., et al. 2003, The Messenger, 114, 20

McClure, R. D. 1984, ApJ, 280, L31

McWilliam, A. 1997, ARA\&A, 35, 503

Mennessier, M. O., Luri, X., Figueras, F., et al. 1997, A\&A, 326, 722

Mermilliod, J.-C. 1996, in The Origins, Evolution and Destinies of Binary Stars in Clusters, ed. E. F. Milone, \& J.-C. Mermilliod (San Francisco: ASP), ASP Conf. Ser., 90, 95

Munari, U., \& Buson, L. M. 1992, A\&A, 255, 158

Munari, U., Yudin, B. F., Taranova, O. G., et al. 1992, A\&AS, 93, 383

Mürset, U., Nussbaumer, H., Schmid, H. M., \& Vogel, M. 1991, A\&A, 248,458

Mürset, U., \& Schmid, H. M. 1999, A\&AS, 137, 473

Musaev, F. A., Galazutdinov, G. A., Sergeev, A. V., Karpov, N. V., \& Podyachaev, Y. V. 1999, Kinematika I Fizika Nebesnyh Tel, 15, 282

Pereira, C. B., \& Porto de Mello, G. F. 1997, AJ, 114, 2128

Pereira, C. B., Smith, V. V., \& Cunha, K. 1998, AJ, 116, 1977

Pinsonneault, M. H., Sneden, C., \& Smith, V. V. 1984, PASP, 96, 239
Piskunov, N. E., Kupka, F., Ryabchikova, T. A., Weiss, W. W., \& Jeffery, C. S. 1995, A\&AS, 112, 525

Pourbaix, D. 2004, in Spectroscopically and Spatially Resolving the Components of Close Binary Stars, ed. R. W. Hilditch, H. Hensberge, \& K. Pavlovski (San Francisco), ASP Conf. Ser., 318, 132

Pourbaix, D., \& Arenou, F. 2001, A\&A, 372, 935

Pourbaix, D., \& Boffin, H. M. J. 2003, A\&A, 398, 1163

Pourbaix, D., \& Jorissen, A. 2000, A\&AS, 145, 161

Pourbaix, D., Tokovinin, A. A., Batten, A. H., et al. 2004, A\&A, 424 727

Preston, G. W., \& Sneden, C. 2001, AJ, 122, 1545

Schaller, G., Schaerer, D., Meynet, G., \& Maeder, A. 1992, A\&AS, 96, 269

Schmid, H. M., \& Nussbaumer, H. 1993, A\&A, 268, 159

Schwarz, H. E. 1991, A\&A, 243, 469

Šleivyte, J., \& Bartkevičius, A. 1990, Vilnius Astronomijos Observatorijos Biuletenis, 85, 3

Smith, V. V., Cunha, K., Jorissen, A., \& Boffin, H. M. J. 1996, A\&A, 315,179

Smith, V. V., Cunha, K., Jorissen, A., \& Boffin, H. M. J. 1997, A\&A, 324,97

Theuns, T., Boffin, H. M. J., \& Jorissen, A. 1996, MNRAS, 280, 1264

Tsymbal, V. 1996, in Model Atmospheres and Spectrum Synthesis, ed. S. J. Adelman, F. Kupka, \& W. W. Weiss (San Francisco: ASP), ASP Conf. Ser., 108, 198

Udry, S., Jorissen, A., Mayor, M., \& Van Eck, S. 1998, A\&AS, 131, 25

Udry, S., Mayor, M., \& Queloz, D. 1999, in Precise Stellar Radial Velocities, ed. J. B. Hearnshaw, \& C. D. Scarfe (San Francisco), ASP Conf. Ser., 170, 367

Van Eck, S., \& Jorissen, A. 2002, A\&A, 396, 599

van Leeuwen, F., \& Evans, D. W. 1998, A\&AS, 130, 157

Vilhu, O., Tuominen, I., Kyrolainen, J., \& Holsti, N. 1977, Obs. Astrophys. Lab. Univ. Helsinki Rep. No. 3

Yamashita, Y., \& Norimoto, Y. 1981, Annals of the Tokyo Astronomical Observatory, 18, 125

Začs, L., Schmidt, M. R., Musaev, F. A., Galazutdinov, G. A., \& Sperauskas, J. 2005, A\&A, 441, 303

Začs, L., Schmidt, M. R., \& Schuster, W. J. 2000, A\&A, 358, 1022 
A. Jorissen et al.: Metal-deficient Ba stars and yellow symbiotics, Online Material $p 1$

\section{Online Material}


A. Jorissen et al.: Metal-deficient Ba stars and yellow symbiotics, Online Material $p 2$

Table 7. Individual CORAVEL radial velocities and associated errors for HIP 34795, HIP 58596, HIP 76605, BD $+3^{\circ} 2688$ and $\mathrm{BD}+04^{\circ} 2466$. The last column indicates which one of the two CORAVEL spectrovelocimeters has been used (NO = CORAVEL north at the Observatoire de Haute Provence; $\mathrm{SO}=\mathrm{CORAVEL}$ south on the Danish 1.54-m telescope at ESO).

\begin{tabular}{|c|c|c|c|c|}
\hline \multicolumn{4}{|c|}{ "HIP 34795 = HD 55496} & \\
\hline DDMMYY & HJD & $\begin{array}{c}\mathrm{RV} \\
\left(\mathrm{km} \mathrm{s}^{-1}\right)\end{array}$ & $\begin{array}{c}\epsilon \\
\left(\mathrm{km} \mathrm{s}^{-1}\right)\end{array}$ & \\
\hline 050183 & 45340.695 & 315.30 & 0.33 & $\mathrm{SO}$ \\
\hline 151283 & 45684.797 & 314.79 & 0.33 & SO \\
\hline 120684 & 45864.434 & 315.71 & 0.46 & $\mathrm{SO}$ \\
\hline 310185 & 46097.658 & 314.78 & 0.34 & SO \\
\hline 271185 & 46397.826 & 314.95 & 0.30 & SO \\
\hline 111186 & 46746.858 & 314.56 & 0.31 & $\mathrm{SO}$ \\
\hline 101286 & 46775.595 & 316.07 & 0.56 & NO \\
\hline 150187 & 46811.682 & 314.98 & 0.31 & SO \\
\hline 160288 & 47208.394 & 316.01 & 0.49 & NO \\
\hline 231088 & 47458.704 & 315.83 & 0.70 & NO \\
\hline 151288 & 47511.857 & 315.11 & 0.33 & $\mathrm{SO}$ \\
\hline 090289 & 47567.393 & 315.39 & 0.56 & NO \\
\hline 241189 & 47855.642 & 315.44 & 0.57 & NO \\
\hline 070390 & 47958.339 & 317.36 & 0.66 & NO \\
\hline 050490 & 47987.497 & 314.78 & 0.31 & $\mathrm{SO}$ \\
\hline 061190 & 48202.697 & 315.63 & 0.45 & $\mathrm{NO}$ \\
\hline 031290 & 48229.645 & 316.10 & 0.56 & NO \\
\hline 180391 & 48334.380 & 314.95 & 0.62 & $\mathrm{NO}$ \\
\hline 110393 & 49058.361 & 315.00 & 0.67 & NO \\
\hline 130393 & 49060.338 & 314.32 & 0.80 & $\mathrm{NO}$ \\
\hline 180194 & 49371.471 & 315.37 & 0.58 & NO \\
\hline 301295 & 50082.752 & 313.99 & 0.34 & SO \\
\hline 231196 & 50411.600 & 316.66 & 0.56 & NO \\
\hline 120197 & 50461.480 & 317.72 & 0.63 & NO \\
\hline \multicolumn{5}{|c|}{ HIP 58596 = HD 104340} \\
\hline DDMMYY & HJD & $\begin{array}{c}\mathrm{RV} \\
\left(\mathrm{km} \mathrm{s}^{-1}\right)\end{array}$ & $\begin{array}{c}\epsilon \\
\left(\mathrm{km} \mathrm{s}^{-1}\right)\end{array}$ & \\
\hline 141289 & 47875.832 & 265.29 & 0.45 & $\mathrm{SO}$ \\
\hline 140392 & 48696.805 & 264.18 & 0.30 & SO \\
\hline 120393 & 49059.755 & 266.40 & 0.29 & SO \\
\hline 190393 & 49066.707 & 264.89 & 0.33 & $\mathrm{SO}$ \\
\hline 050493 & 49083.708 & 265.41 & 0.28 & SO \\
\hline 200493 & 49098.453 & 263.93 & 0.46 & NO \\
\hline 080394 & 49420.525 & 263.34 & 0.47 & $\mathrm{NO}$ \\
\hline 030494 & 49446.435 & 266.38 & 0.41 & NO \\
\hline 140594 & 49487.360 & 265.37 & 0.46 & NO \\
\hline 260195 & 49744.638 & 264.59 & 0.56 & NO \\
\hline 300395 & 49807.475 & 263.16 & 0.52 & NO \\
\hline 210795 & 49920.516 & 264.34 & 0.30 & $\mathrm{SO}$ \\
\hline 191295 & 50071.721 & 265.60 & 0.37 & NO \\
\hline 150296 & 50129.556 & 263.70 & 0.43 & $\mathrm{NO}$ \\
\hline 130197 & 50462.684 & 265.61 & 0.45 & NO \\
\hline \multicolumn{5}{|c|}{ HIP 76605 = HD 139409} \\
\hline DDMMYY & HJD & $\begin{array}{c}\mathrm{RV} \\
\left(\mathrm{km} \mathrm{s}^{-1}\right)\end{array}$ & $\begin{array}{c}\epsilon \\
\left(\mathrm{km} \mathrm{s}^{-1}\right)\end{array}$ & \\
\hline 090790 & 48082.629 & 62.43 & 0.30 & $\mathrm{SO}$ \\
\hline 260794 & 49560.513 & 63.36 & 0.27 & $\mathrm{SO}$ \\
\hline
\end{tabular}

Table 7. continued.

\begin{tabular}{|c|c|c|c|c|}
\hline \multicolumn{4}{|c|}{ HIP $55852=$ BD +04 2466} & \\
\hline DDMMYY & HJD & $\begin{array}{c}\mathrm{RV} \\
\left(\mathrm{km} \mathrm{s}^{-1}\right)\end{array}$ & $\begin{array}{c}\epsilon \\
\left(\mathrm{km} \mathrm{s}^{-1}\right)\end{array}$ & \\
\hline 070282 & 45008.814 & 38.11 & 0.69 & $\mathrm{SO}$ \\
\hline 130684 & 45865.491 & 44.73 & 0.74 & SO \\
\hline 030285 & 46100.832 & 45.14 & 0.68 & $\mathrm{SO}$ \\
\hline 290385 & 46154.675 & 44.78 & 0.58 & $\mathrm{SO}$ \\
\hline 200186 & 46451.831 & 43.54 & 0.53 & SO \\
\hline 140286 & 46476.783 & 43.32 & 0.49 & $\mathrm{SO}$ \\
\hline 291186 & 46764.706 & 42.05 & 0.96 & NO \\
\hline 090187 & 46805.864 & 41.36 & 0.49 & SO \\
\hline 080287 & 46835.808 & 41.99 & 0.41 & SO \\
\hline 280188 & 47189.812 & 40.50 & 0.43 & SO \\
\hline 040388 & 47225.726 & 39.83 & 0.41 & SO \\
\hline 250488 & 47277.657 & 40.17 & 0.48 & $\mathrm{SO}$ \\
\hline 051288 & 47501.707 & 37.84 & 0.69 & NO \\
\hline 060389 & 47592.565 & 38.14 & 0.64 & NO \\
\hline 220589 & 47669.526 & 37.64 & 0.49 & $\mathrm{SO}$ \\
\hline 241289 & 47885.857 & 37.48 & 0.45 & SO \\
\hline 050290 & 47928.800 & 36.83 & 0.48 & SO \\
\hline 160290 & 47939.743 & 37.05 & 0.43 & $\mathrm{SO}$ \\
\hline 260290 & 47949.595 & 37.00 & 0.69 & NO \\
\hline 040490 & 47986.631 & 37.01 & 0.42 & $\mathrm{SO}$ \\
\hline 110490 & 47993.594 & 35.99 & 0.49 & SO \\
\hline 050590 & 48017.647 & 36.35 & 0.61 & $\mathrm{SO}$ \\
\hline 020690 & 48045.501 & 36.82 & 0.46 & SO \\
\hline 260291 & 48314.487 & 34.56 & 0.73 & NO \\
\hline 260391 & 48342.724 & 34.92 & 0.47 & SO \\
\hline 150392 & 48697.747 & 34.26 & 0.42 & SO \\
\hline 120393 & 49059.726 & 33.77 & 0.39 & SO \\
\hline 070493 & 49085.670 & 33.15 & 0.40 & SO \\
\hline 080493 & 49086.448 & 32.60 & 0.70 & NO \\
\hline 100294 & 49394.609 & 34.54 & 0.77 & NO \\
\hline 020394 & 49414.713 & 35.84 & 0.43 & $\mathrm{SO}$ \\
\hline 070394 & 49419.515 & 34.92 & 0.52 & $\mathrm{NO}$ \\
\hline 080494 & 49451.424 & 37.51 & 0.88 & NO \\
\hline 140594 & 49487.351 & 36.78 & 0.64 & NO \\
\hline 310195 & 49749.592 & 39.24 & 0.74 & NO \\
\hline 150395 & 49792.681 & 41.24 & 0.44 & SO \\
\hline 020495 & 49810.460 & 40.55 & 0.44 & NO \\
\hline 190795 & 49918.506 & 41.94 & 0.53 & SO \\
\hline 191295 & 50071.715 & 43.32 & 0.60 & NO \\
\hline 130197 & 50462.674 & 44.33 & 0.90 & NO \\
\hline 200199 & 51199.634 & 41.42 & 0.80 & NO \\
\hline \multicolumn{5}{|c|}{$\mathrm{BD}+03^{\circ} 2688$} \\
\hline DDMMYY & HJD & $\begin{array}{c}\mathrm{RV} \\
\left(\mathrm{km} \mathrm{s}^{-1}\right)\end{array}$ & $\begin{array}{c}\epsilon \\
\left(\mathrm{km} \mathrm{s}^{-1}\right)\end{array}$ & \\
\hline 080493 & 49086.481 & 33.16 & 0.43 & $\mathrm{NO}$ \\
\hline 050394 & 49417.506 & 32.35 & 0.47 & NO \\
\hline 030494 & 49446.510 & 32.33 & 0.44 & $\mathrm{NO}$ \\
\hline 140594 & 49487.388 & 33.00 & 0.47 & NO \\
\hline 030295 & 49752.658 & 33.52 & 0.52 & NO \\
\hline 300395 & 49807.506 & 32.64 & 0.71 & NO \\
\hline 201295 & 50072.729 & 33.07 & 0.46 & NO \\
\hline
\end{tabular}

